\title{
Hrq1/RECQL4 regulation is critical for preventing aberrant recombination during DNA intrastrand crosslink repair and is upregulated in breast cancer
}

Thong T. Luong ${ }^{1}$, Zheqi Li $^{2}$, Nolan Priedigkeit ${ }^{2}$, Phoebe S. Parker ${ }^{1}$, Stefanie Böhm ${ }^{1}$, Kyle

Rapchak $^{1}$, Adrian V. Lee ${ }^{2}$, Kara A. Bernstein ${ }^{1, \pm}$

${ }^{1}$ University of Pittsburgh School of Medicine, Department of Pharmacology and Chemical

Biology, UPMC Hillman Cancer Center, 5117 Centre Avenue, Pittsburgh, Pennsylvania, USA.

${ }^{2}$ Women's Cancer Research Center, UPMC Hillman Cancer Center, Magee-Womens Research

Institute, 204 Craft Avenue, Pittsburgh, PA 15213

${ }^{ \pm}$Corresponding Author: Kara A. Bernstein, karab@pitt.edu 


\begin{abstract}
Human RECQL4 is a member of the RecQ family of DNA helicases and functions during DNA replication and repair. RECQL4 mutations are associated with developmental defects and cancer. Although RECQL4 mutations lead to disease, RECQL4 overexpression is also observed in cancer, including breast and prostate. Thus, tight regulation of RECQL4 protein levels is crucial for genome stability. Because mammalian RECQL4 is essential, how cells regulate RECQL4 protein levels is largely unknown. Utilizing budding yeast, we investigated the RECQL4 homolog, $H R Q 1$, during DNA crosslink repair. We find that Hrq1 functions in the error-free template switching pathway to mediate DNA intrastrand crosslink repair. Although Hrq1 repairs cisplatin-induced lesions, it is paradoxically degraded by the proteasome following cisplatin treatment. By identifying the targeted lysine residues, we show that preventing Hrq1 degradation results in increased recombination and mutagenesis. Like yeast, human RECQL4 is similarly degraded upon exposure to crosslinking agents. Furthermore, over-expression of RECQL4 results in increased RAD51 foci, which is dependent on its helicase activity. Using bioinformatic analysis, we observe that $R E C Q L 4$ overexpression correlates with increased recombination and mutations. Overall, our study uncovers a role for Hrq1/RECQL4 in DNA intrastrand crosslink repair and provides further insight how misregulation of RECQL4 can promote genomic instability, a cancer hallmark.
\end{abstract}




\section{Introduction}

Accurate and timely repair of DNA damage is critical for genomic integrity and human health. Disruptions of DNA repair genes are frequently associated with diseases such as cancer and aging. One such gene is RECQL4, which belongs to the evolutionarily conserved family of RecQ helicases. This family of 3' to 5'- DNA helicases is often referred to as the "Guardians of the Genome" due to crucial roles in DNA recombination, replication, and repair that are conserved from yeast to man (1-5). Mutations in RECQL4 are associated with three heritable autosomal diseases: Rothmund-Thomson syndrome (RTS type II), Baller-Gerold syndrome (BGS), and RAPADILINO, each characterized by developmental defects, cancer and/or premature aging (6-12). Although RECQL4 dysfunction is associated with hereditable diseases, recent studies have shown that overexpression of RECQL4 is linked to multiple cancer types such as breast, hepatic, gastric, and prostate. In each cancer type, high levels of RECQL4 are correlated with poor prognosis (13-16). Since both inactivation or overexpression of $R E C Q L 4$ cause human disease and genetic instability, RECQL4 protein levels must be tightly regulated.

Although RECQL4 is critical for genome integrity and disease prevention, previous studies of mammalian RECQL4 were stymied due to technical difficulties, including the embryonic lethality of mouse knockout models and the inviability of human RECQL4 knockout cell lines (8, 17-19). In 2008, HRQ1, was discovered as the Saccharomyces cerevisiae homolog of RECQL4 (20). Since HRQ1 is non-essential, yeast is a valuable model to elucidate $R E C Q L 4$ gene family function. For example, analysis of $h r q 1 \Delta$ cells identified a novel function for Hrq1 in DNA crosslink repair $(21,22)$. HRQ1-disrupted cells are sensitive to DNA crosslinking agents such as cisplatin and mitomycin $\mathrm{C}(\mathrm{MMC})$, which predominantly create intrastrand or interstrand 
crosslinks, respectively. Furthermore, recent studies suggest that human RECQL4 also has a role in crosslink repair since RECQL4 shRNA knockdown leads to cisplatin sensitivity in a triplenegative breast cancer (TNBC) cell line (14). Although the RECQL4 gene family has a conserved role in crosslink repair, its molecular function during this process has yet to be elucidated.

DNA crosslinking agents can induce two types of damage including, interstrand crosslinks (ICL) and intrastrand crosslinks. In ICLs, the Watson and Crick strands are covalently linked, whereas in an intrastrand crosslink, the same DNA strand is covalently linked to itself (23-26). Due to the different nature of the crosslinks, the mechanism of how these two adducts are repaired is also unique. While there are some key differences in how ICLs are repaired from yeast to man, during replication-coupled ICL repair, the steps are largely conserved (27-31). Following damage recognition, endonucleases nick either side of the damaged DNA, which then mediates exonuclease to come in and degrade the damaged DNA. This "unhooking" step results in a ssDNA gap. This gap may be filled in by the post-replicative repair (PRR) pathway $(28,32-$ 35). The PRR pathway consists of an error-prone and error-free branch. Utilization of the different branches is mediated by PCNA (Pol30 in yeast) ubiquitylation. For example, in both yeast and humans mono-ubiquitylation of PCNA at lysine 164 (K164) recruits error-prone translesion synthesis polymerases to fill the gap (36). On the other hand, polyubiquitylation of the same K164 residue on PCNA results in error-free homology-directed repair (37). In contrast to ICLs, intrastrand crosslinks are primarily repaired by the nucleotide excision repair (NER) pathway (38-41). However, if the replication fork encounters the intrastrand crosslink and stalls, then the PRR and DNA damage tolerance pathways can mediate bypass of this adduct using the 
mechanism described above $(36,37,42,43)$. Subsequently NER, will excise and degrade the damaged DNA and the gap with be filled using DNA polymerases and ligases.

Recent studies demonstrate that Hrq1 functions during NER to repair cisplatin-induced DNA lesions (44-47). However, there are conflicting studies as to whether Hrql also has a role in PRR (44-46). For example, a study in A. thaliana suggests that Hrq1 functions in PRR while two independent studies in $S$. pombe and $S$. cerevisiae, respectively, suggest that Hrq1 functions independently of PRR (44-46). Therefore, it remains controversial as to whether Hrq1 or its mammalian homology, RECQL4, truly functions during PRR.

Here, similar to plants and in contrast to previous studies in budding and fission yeast, we find that the budding yeast Hrq1 has a role in the error-free branch of PRR specifically during intrastrand crosslink repair. Furthermore, we performed genetic analysis that suggests that human RECQL4 also functions during PRR. Paradoxically, despite a conserved role in cisplatin resistance, Hrq1 is degraded by the proteasome following cisplatin exposure. We find that $H R Q 1$ overexpression or its stabilization leads to increased recombination and mutation rates. Furthermore, suggesting a conserved regulatory mechanism, we show that endogenous human RECQL4 protein levels decrease following cisplatin and acetaldehyde treatment. Indicative of increased recombination, overexpression of $R E C Q L 4$ results in increased RAD51 foci, which is dependent on its helicase activity. Lastly, bioinformatic analysis reveals that high levels of RECQL4 are correlated with increased tumor mutation burden. Importantly, we observe that in TNBC, the protein levels of RECQL4 are a predictive marker to cisplatin response. Our work uncovers a role for Hrq1 in the error-free branch PRR repair and a conserved regulatory 
mechanism between yeast Hrq1 and mammalian RECQL4 following DNA intrastrand crosslink damage.

\section{Materials and Methods}

\section{Yeast strains}

The yeast strains used in this study are listed in Supplemental Table 1. All strains are isogenic to W303 $(48,49)$. Yeast media and plates were prepared as previously described (50). Strain construction for knockouts and epitope tagging was performed as described in (51). After transformation and selection, knockouts were verified by sequencing using primers that flank each gene; western blot or fluorescent microscopy was used to confirmed epitope tagging.

\section{Mammalian cell culture}

U2OS cells (ATCC) were cultured in DMEM w/ 10\% FBS and 1x penicillin-streptomycin (Life Technologies: $50 \mathrm{u} / \mathrm{ml}$ penicillin, $50 \mu \mathrm{g} / \mathrm{ml}$ streptomycin) at $5 \% \mathrm{CO}_{2}$.

\section{Serial Dilutions, cisplatin and mitomycin $\mathrm{C}$ plates}

The indicated cultures were grown in $4 \mathrm{ml} \mathrm{SC}$ medium ( $\mathrm{pH}$ 5.8) for cisplatin exposure or YPD for MMC exposure overnight at $30^{\circ} \mathrm{C}$, and subsequently diluted to $0.2 \mathrm{OD}_{600}$ and grown for 2.5 hours until the cultures reached logarithm phase. The cultures were five-fold serially diluted starting at $0.2 \mathrm{OD}_{600}$ and $5 \mu 1$ were spotted on the indicated plates and grown for 2 days at $30^{\circ} \mathrm{C}$ before being photographed. Photoshop was used to crop and enhance the contrast of the images, and subsequently converted to black and white images in the Figures. 
Cisplatin (SelleckChem S1166) was dissolved to $100 \mathrm{mg} / \mathrm{ml}$ stock solution in dimethyl sulfoxide (DMSO). Synthetic complete (SC) plates containing the indicated doses of cisplatin, were made by diluting the stock solution. Cisplatin was handled in the dark and plates were stored in the dark and used within 24 hours.

MMC (SelleckChem S8146) was dissolved to $50 \mathrm{mg} / \mathrm{ml}$ stock solution in dimethyl sulfoxide (DMSO). YPD plates containing the indicated doses of MMC, were made by diluting the stock solution. MMC was handled in the dark and plates were stored in the dark and used within 24 hours.

\section{Cycloheximide chase}

Cycloheximide chases in yeast were adapted from (52). Yeast cultures were grown in $3 \mathrm{ml} \mathrm{SC}$ medium ( $\mathrm{pH} 5.8$ ) or YPD overnight at $30^{\circ} \mathrm{C}$, and subsequently diluted to $0.2 \mathrm{OD}_{600}$ in $35 \mathrm{ml}$ $\mathrm{SC} / Y P D$ medium and grown until the cultures reached logarithm phase. The translation inhibitor, cycloheximide (Sigma-Aldrich), was then added to a final concentration of $0.5 \mathrm{mg} / \mathrm{ml}$. In addition to cycloheximide, DMSO $(0.1 \%)$ or cisplatin $(100 \mu \mathrm{g} / \mathrm{ml})$ or MMC $(100 \mu \mathrm{g} / \mathrm{ml})$ diluted in DMSO was added to the culture and grown at $30^{\circ} \mathrm{C}$ for the indicated time points. Where indicated in the text, cells were pretreated with proteasome inhibitor MG-132 $(50 \mu \mathrm{M}$, SelleckChem S2619) for one hour prior to CHX addition. Equal amounts of cells (0.75 OD 600$)$ were taken at each time-point, pelleted, supernatant was removed, and washed once with dd $\mathrm{H} 2 \mathrm{O}$. The pellets were flash frozen on dry ice. Protein was extracted from whole cell lysates by TCA preparation as described in $51 \mu$ l of loading buffer (53). The $13 \mu 1$ of protein was run on a $10 \%$ SDS-PAGE gel and transferred to a PDVF membrane by semidry transfer (Bio-Rad) at 13V for 2 
hours 15 min. Western blot using Myc antibodies were used detect Hrq1-9Myc ( $\alpha$-Myc, mouse monoclonal, Santa Cruz Biotechnology, 1:500) or Kar2 ( $\alpha$-Kar2, rabbit polyclonal, Santa Cruz biotechnology, 1:5000) as a loading control.

Mammalian CHX chases were adapted from the yeast protocol and performed using U2OS cells. Cells were seeded into 6-well plates at 75,000 cells per well and were grown for three days in 10\% FBS-DMEM. The cells were then treated with $\mathrm{CHX}(50 \mu \mathrm{g} / \mathrm{ml})$ and untreated, or $50 \mu \mathrm{M}$ cisplatin (diluted in water), or $75 \mu \mathrm{M}$ acetaldehyde (Sigma-Aldrich). One well of each 6-well dish per time-point was collected and lysed in RIPA buffer [150 mM NaCl, $50 \mathrm{mM}$ Tris-HCL pH 7.2, 1\% Triton X-100, 0.5 Na-deoxycholate, 0.1\% SDS, 1mM EDTA, 1x Pierce Protease Inhibitor (Thermo), 1x Phosphatase Inhibitor (Thermo)]. Protein concentration was measured via Bradford assay, equal amount of protein were run on a 10\% SDS-PAGE gel, transferred to PDVF membrane, and blotted endogenous RECQL4 (rabbit polyclonal, Cell Signaling, 1:500, WB) and $\gamma$-tubulin ((mouse monoclonal, Sigma Aldrich, 1:5000) or GAPDH (mouse monoclonal, UBP Bio, 1:1000) as a loading control. The blots were scanned, the images were cropped and grayscale using Photoshop. ImageJ was used to determine relative protein quantity by mean gray value measurement. It was then normalized to the loading control and to timepoint zero.

\section{Cell Cycle Analysis}

Cells expressing Hrq1-9myc were grown overnight in $4 \mathrm{ml} \mathrm{YPD}$ at RT, and subsequently diluted to $0.2 \mathrm{OD}_{600}$ in $30 \mathrm{ml} \mathrm{YPD}$ and grown to logarithmic phase at RT. Alpha factor (Genescript) diluted in DMSO was added to a final concentration of $10 \mu \mathrm{M}$, and cells were arrested in $\mathrm{G} 1$ for 
3 hours. The asynchronous control sample was removed directly prior to alpha factor addition.

To release the cells from alpha factor, the yeast was pelleted, washed twice in an equal volume of water, and transferred into $30 \mathrm{~mL}$ YPD medium. Samples of equal amounts of cells were taken every 20-minutes for FACS and protein analysis. Protein samples were prepared as described above, with the exception that $\mathrm{Clb} 2$ (G2/M cyclin) was also analyzed (rabbit polyclonal, Santa Cruz Biotechnology, 1:2000). FACS samples were pelleted and washed once with water before being fixed with $70 \% \mathrm{EtOH}$ and stored in the dark at $4^{\circ} \mathrm{C}$. Subsequently, the samples were treated with protease and RNase and DNA was stained with propidium iodide and analyzed by FACS as described (54).

\section{Ubiquitin Pulldown}

Ubiquitin pulldown protocol was modified from the Tansey’s Lab $(55,56)$. Briefly, pdr5 4 Hrq19myc and pdr5 4 Hrq1-7KR-6xHA strains were transformed with YEpHisUbStu, which expresses 6x-His-Ubiquitin under the CUP1 promoter. Cells were grown in SC-TRP medium to select for the YEpHisUbStu and grown to logarithmic phase. Expression of 6x-His-Ubiquitin expression was induced by addition of $500 \mu \mathrm{M} \mathrm{CuSO}_{4}$. After 2.5 hours of growth, MG-132 (50 $\mu \mathrm{M})$ was added then grown for another 45 minutes before addition of DMSO $(0.1 \%)$ or cisplatin $(100 \mu \mathrm{g} / \mathrm{ml})$ and further grown for 45 minutes before being pelleted and frozen. The cell pellets were lysed using glass beads and ubiquitylated proteins were pulldown by affinity purification using Ni-NTA beads (ThermoFisher \#88221). The Ni-NTA beads were then washed 3 times with $10 \mathrm{mM}$, followed by 3 times with $17.5 \mathrm{mM}$, then finally 1 time with $20 \mathrm{mM}$ imidazole. The protein bound to the beads was eluted in $50 \mathrm{ul}$ of $2 \mathrm{x}$ Laemmli buffer with $0.8 \mathrm{M}$ imidazole. The entire sample was loaded on $1.5 \mathrm{~mm} \mathrm{8 \%} \mathrm{SDS-PAGE} \mathrm{gel} \mathrm{and} \mathrm{ran} \mathrm{at} 85$ volts for 2 hours and 
transferred to PDVF membrane via semidry transfer (Bio-Rad) at $19 \mathrm{~V}$ for 2 hours and 30 minutes. We probed using $\alpha$-Myc (1:500 Santa Cruz) or $\alpha$-HA (1:5000 Abcam) to detect ubiquitylation status of Hrq1-9xMyc or Hr1-7KR-6xHA. For each condition, we utilized densitometry (ImageJ) to quantify ubiquitylation amount by normalizing ubiquitylated band to the input. Photoshop was used to crop and grayscale the image.

\section{Protein Immunoprecipitation}

Hrq1-9myc $p d r 5 \Delta$ or Hrq1-7KR-6xHA $p d r 5 \Delta$ strains were grown overnight in $3 \mathrm{~mL}$ of YPD. Subsequently, the cultures were diluted to $0.2 \mathrm{OD}_{600}$ in $100 \mathrm{ml}$ of YPD. After 2 hours, MG-132 $(50 \mu \mathrm{M})$ was added for 45 minutes before addition of DMSO $(0.1 \%)$ or cisplatin $(100 \mu \mathrm{g} / \mathrm{ml})$ for another 45 minutes before the cells were pelleted and frozen. The cell pellets were resuspended in lysis buffer [50 mM Tris, $100 \mathrm{mM} \mathrm{NaCl}, 2 \mathrm{mM} \mathrm{MgCl}$, 10\% glycerol, $0.1 \%$ NP-40, 1x Pierce Protease Inhibitor (Thermo), 1x Phosphatase Inhibitor (Thermo), 2 mM PMSF]. Glass beads were added, and the mixture was vortex for 5 minutes at $4^{\circ} \mathrm{C}$. Next, $31.5 \mu 1$ of $10 \%$ NP-40 was added and the mixture incubated on ice for 5 minutes. Afterwards, it was spun down at 5,000xg for 5 minutes at $4^{\circ} \mathrm{C} .15 \mu \mathrm{l}$ of the supernatants were set aside for the input and $15 \mu$ l of loading buffer was added. The remaining $250 \mu 1$ of the sample was incubated with $20 \mu 1$ of washed magnetic Myc or HA beads at $4^{\circ} \mathrm{C}$ for 3 hours on a rotator. The beads were washed four times with lysis buffer with 5 minutes incubations on ice between washes. Following the final wash, all the supernatants were removed and $25 \mu 1$ of loading buffer was added to the beads. The samples were boiled at $65^{\circ} \mathrm{C}$ for 10 minutes and then loaded on $10 \%$ SDS-PAGE gel and ran at 90 volts for 2 hours and transferred to a PDVF membrane via semidry transfer (Bio-Rad) at $13 \mathrm{~V}$ for 3 hours. The blots were probed using $\alpha$-Myc (1:500 Santa Cruz) or $\alpha-H A$ (1:5000 Abcam) 
antibodies to detect Hrq1 and $\alpha$-ubiquitin (1:1000 Santa Cruz) antibodies to detect ubiquitylated Hrq1-9xMyc or Hr1-7KR-6xHA. For each condition, we utilized densitometry (ImageJ) to quantify ubiquitylation amount by normalizing ubiquitylated band to the amount of Hrq1 pulleddown. Photoshop was used to crop and grayscale the image.

\section{Canavanine Mutagenesis Assay}

Mutagenesis assay protocol was adapted from Godin et al. 2016 (57). Two individual colonies of wild-type (WT), Hrq1-7KR, Hrq1-OE were grown in $3 \mathrm{ml}$ of SC with 2\% galactose, to induce Hrq1-overexpression, either with or without cisplatin overnight at $30^{\circ} \mathrm{C}$ to saturation.

Subsequently, the culture was diluted to $2 \mathrm{OD}_{600}$ and $200 \mu 1$ was plated onto SC-ARG+CAN plate or further diluted 100,000-fold and $200 \mu \mathrm{l}$ was plated onto SC plate (these plates were made with galactose). The plates were incubated for 3 days at $30^{\circ} \mathrm{C}$. Plates were scanned and colonies were counted using OpenCFU software (58). Mutation rate was calculated by counting colonies that grew on SC-ARG+CAN vs total colonies that grew on SC. For each trial, a mean rate was calculated and graphed alongside the median from multiple experiments. MannWhitney test was used to determined significance.

\section{Direct Repeat Recombination Assays}

Direct repeat recombination assays were performed as described (59) except that the cultures were grown overnight in $\mathrm{SC}$ with $2 \%$ galactose either with or without cisplatin. In this assay, two leu2 heteroalleles are disrupted with EcoRI and BsteII, respectively, with an intervening URA3 gene. Total recombination is measure by restoration of a function $L E U 2$ gene, which allows yeast to grow on plates that lack leucine. The experiment was done in nonuplicate and repeated. 
Recombination rate was calculated and graphed alongside the median from the two experiments. Mann-Whitney test was used to determined significance.

\section{Cell Proliferation (MTS) Assay}

Cells were seeded at 50,000/well in a 6 well plate. The next day $30 \mathrm{nM}$ of siRNAs were transfected using INTERFERin (VWR \#89129-930) per manufacturer’s instructions. After 48 hours, the cells were then seeded 5,000 cells/well in a 96-well plate, the remaining cells were used for western to confirm protein knockdown levels. The next morning, cells were treated with the indicated concentration of cisplatin. After 72 hours of treatment, 20 ul of MTS reagent was added to the media and allowed to incubate in the dark for two hours. Growth was measured by absorbance at $490 \mathrm{~nm}$.

\section{Clonogenic Survival Assay}

Initial seeding and siRNAs transfection were performed as described for the MTS assays, except that after the 48 hours of siRNA treatment, the cells were then seeded 250 cells/well in a 6 -well plate. The next morning, cells were treated with the indicated concentration of cisplatin. After 24 hours of treatment, cells were washed with PBS and fresh medium was added. Colonies were grown for 17 days. For staining, cells were washed twice with PBS before being fixed with methanol for 20 minutes and stained with crystal violet solution $(0.5 \%$ crystal violet, $20 \%$ methanol) for 30 minutes. Colonies were counted using ImageJ plugin, ColonyArea (60). Each condition was plated in triplicate and the experiment was independently performed three times. Results are plotted as a normalized area, where in the treated conditions were normalized to the untreated conditions. 


\section{Immunofluorescence}

U2OS cells were seeded at 50,000 cells/well in a 6-well dish that contained a coverslip in each well. The next day, the cells were transfected with an empty plasmid, plasmid expressing RECQL4 or RECQL4-K508A under CMV promoter. 72 hours following transfection, the cells were treated with or without cisplatin for one hour in the dark. Afterwards, the cells were washed with PBS and fresh medium was added. The treated cells were allowed to recover for 2 hours. The coverslip was then removed and fixed with 4\% PFA for 20 minutes in the dark at $4^{\circ} \mathrm{C}$. After fixation, the sample was treated with extraction buffer and then washed and incubated overnight with $\alpha$-RAD51 (1:2000). Subsequently, the samples were washed and then incubated with Goat anti-Rabbit IgG Alexa Fluor 488 (1:2000) for an hour at RT. Following secondary antibody incubation, the samples were washed then mounted onto a slide using Prolong Gold solution with DAPI. The slides were allowed to dry overnight before imaging. Foci were image with Nikon-TiE, RAD51 foci was quantified using ImageJ plug-in, Fiji.

\section{Neutral comet assay to detect ssDNA}

Initial seeding and transfection and cisplatin treatment are the same as described for the immunofluorescence experiments with the exception that a coverslip was not added. Following the 2 hours recovery, the cells were trypsinized and washed before being resuspended in PBS at 100,000 cells per milliliter. Subsequently, the cells were combined with molten LMAgarose

(Cat\# 4250-050-02) at 1:10 (cells: agarose) ratio, and immediately $30 \mu \mathrm{l}$ was pipetted onto two 20-well CometSlideTM (Cat\# 4252-02K-01). The mixture solidified at $4^{\circ} \mathrm{C}$ before being immersed in chilled lysis solution (Cat\# 4250-050-01) overnight. Subsequently, the slides were 
washed three times with 1x S1 nuclease buffer $(50 \mathrm{mM} \mathrm{NaCl}, 30 \mathrm{mM}$ sodium acetate, $\mathrm{pH} 4.6$ and 5\% glycerol). S1 nuclease (ThermoFisher \#18001016) at $20 \mathrm{U} / \mathrm{ml}$ in S1 nuclease buffer and $50 \mathrm{mM} \mathrm{NaCl}$ was added to one of the two slides and incubated for $30 \mathrm{~min}$ at $37^{\circ} \mathrm{C}$. As a control, the other slide was incubated with the same solution, but without S1 addition. The slides were then washed three times in chilled neutral electrophoresis buffer (100 mM Tris base, $300 \mathrm{mM}$ sodium acetate). Afterwards, the slides equilibrated in the neutral electrophoresis buffer for 30 minutes at $4^{\circ} \mathrm{C}$. The slides were then placed in a chilled electrophoresis unit (Cat\# 4250-05-ES) and submerged with chilled electrophoresis buffer. Electrophoresis was then run at 21 volts for 45 minutes at room temperature (RT). After electrophoresis, the slides were transferred to DNA precipitation buffer $(6.7 \mathrm{ml}$ of $7.5 \mathrm{M}$ ammonium acetate and $43.3 \mathrm{ml} \mathrm{95 \%} \mathrm{EtOH})$ and incubated at RT for 30 minutes. Following DNA precipitation, the slides were immersed in $70 \% \mathrm{EtOH}$ and incubated for 30 minutes at RT. The slides were then allowed to dry overnight at RT and subsequently stained using SYBR Gold solution (1 ul of SYBR in $30 \mathrm{ml}$ Tris-EDTA buffer, pH 7.5) for 30 minutes at RT. Afterwards, excess SYBR solution was removed, and the slides were dried overnight at RT. Comets were imaged with Nikon-TiE and quantified using Comet Assay IV software by Instem.

\section{Bioinformatic analysis}

$\mathrm{R}$ version 3.6.1. was used for all the bioinformatic analysis. RECQL4 expression in different samples was determined by first transcriptomic data from TCGA and METABRIC. TCGA data were downloaded from GSE62944 and Log2 (TPM+1) values were used for downstream analysis. For the METABRIC data set, normalized probe intensity values were obtained from 
Synapse (Syn1688369). For genes with multiple probes, probes with the highest inter-quartile range (IQR) were selected to represent the gene.

Tumor mutation burden (TMB) calculation was performed as previous described. Briefly, TCGA mutation annotation files from 982 patients were downloaded from FireBrowse and mutation subtypes were summarized using "maftools" package. Mutations subtypes were classified into truncated (nonsense, frame-shift deletion, frame-shift insertion, splice-site) and non-truncated mutations (missense, in-frame deletion, in-frame insertion, nonstop). TMB was calculated as $2 \mathrm{X}$ Truncating mutation numbers + non-truncating mutation numbers.

Pathway enrichment was performed by gene set variation analysis (GSVA). Using the GSVA program, we inputted the TNBC transcriptomic data from TCGA and METABRIC, and we used the defined 50 Hallmark gene sets from molecular signature database (MSigDB) as our gene sets. The program then provided which pathways and genes were enriched, we then stratified the results based on RECQL4 expression. Clinical and pathological outcome was based on analysis from a (Silver et al. 2010) study where cisplatin was given as a neoadjuvant therapy in 24 TNBC tumors. Transcriptomic data were downloaded from GSE18864. Clinical response to cisplatin was measured as progressive disease (PD), stable disease (SD), partial response (cPR), complete response (cCR). For clinical response, good and poor responders were defined as patients with $\mathrm{cPR} / \mathrm{cCR}$ status and $\mathrm{PD} / \mathrm{SD}$ status respectively. Pathological response to cisplatin was monitored by Miller Payne metric score (grade 1 is no significant tumor reduction and grade 5 is compete tumor reduction). Good and poor responders were defined as patients with a Miller Payne Score of 4-5 or 0-3 respectively. 


\section{Antibodies}

The following primary and secondary antibodies were used at the indicated dilutions: Myc

(mouse monoclonal, Santa Cruz Biotechnology \#sc-40, 1:500, WB); Clb2 (rabbit polyclonal, Santa Cruz Biotechnology \#sc-9071, 1:2000, WB); Kar2 (rabbit polyclonal, Santa Cruz

Biotechnology \#sc-33630, 1:5000, WB); RECQL4 (rabbit polyclonal, Cell Signaling \#2814, 1:500, WB), $\gamma$ - tubulin (mouse monoclonal, Sigma Aldrich \#T5326, 1:5000, WB); GAPDH (mouse monoclonal, UBP Bio \#Y1040, 1:1000, WB); REV1 (mouse monoclonal, Santa Cruz Biotechnology \#sc-393022); RAD51 (rabbit polyclonal, Abcam \#ab63801, 1:2000, IF); AntiMouse HRP (Jackson ImmunoResearch Laboratories \#115-035-003, 1:10000, WB); Anti-Rabbit (Jackson ImmunoResearch Laboratories \#111-035-003, 1:10000, WB), Goat anti-Rabbit IgG Alexa Fluor 488 (ThermoFisher \#A-11034, 1:2000, IF).

\section{Plasmid and oligonucleotides}

The plasmids and oligonucleotides used in this study is listed in Supplemental Table 1 and 2 respectively.

\section{Results}

\section{Hrq1 is degraded by the proteasome upon cisplatin treatment}

Previous reports demonstrate that Hrq1 is important during DNA crosslink repair $(21,22)$. Consistent with prior studies $(21,22)$, we observe that $h r q 1 \Delta$ cells are cisplatin sensitive compared to wild-type (WT) cells (Figure 1a). Since Hrq1 is needed for resistance to DNA crosslinking agents, we reasoned that Hrq1 protein levels may increase upon cisplatin exposure. To address this, we 9xMyc tagged Hrq1 on its C-terminus at its endogenous locus and promoter. 
We verified that 9xMyc-Hrq1 was functional upon exposure of the HRQ1-9myc yeast strain to cisplatin by comparing its growth to the parental WT strain (Supplemental Figure 1a). Although Hrq1 is needed for cisplatin resistance, we paradoxically observe that Hrq1 steady state levels significantly decrease upon cisplatin exposure (Figure 1b). Importantly, Hrq1 degradation is specific to cisplatin since other types of DNA damage, such as methyl methanesulfonate (MMS; an alkylating agent), ionizing radiation (IR; which induces double-strand breaks), or hydroxyurea (HU; which depletes dNTP pools), have no detectible effect on Hrq1 steady state levels (Figure 1b). These results suggest that the reduced Hrq1 protein levels observed are specific to cisplatin.

Next, we sought to determine if the reduced Hrq1 protein levels observed in cisplatin-treated cells are due to protein degradation. To measure Hrq1 protein stability, we performed cycloheximide chase experiments and analyzed cells for Hrq1 protein every 20 minutes for 120 minutes following cycloheximide addition (Figure 1c). Consistent with our previous observations, cisplatin treatment led to a decrease in Hrq1 protein levels, where approximately $50 \%$ of Hrq1 remains 30 minutes following cycloheximide addition, whereas in DMSO treatment more than 50\% of Hrq1 still remains at 120-minute mark (Figure 1c).

Proteasomal degradation is an important regulatory mechanism to ensure proper and timely DNA repair for many DNA repair proteins (61). Therefore, one possibility is that Hrq1 protein levels are regulated by the $26 \mathrm{~S}$ ubiquitin proteasome system (UPS) following DNA damage. To determine whether Hrq1 is degraded by the UPS following cisplatin treatment, we performed cycloheximide chase experiments in the presence of the proteasome inhibitor, MG-132 (Figure 
1d). Hrq1 protein levels were stabilized following cisplatin exposure when the UPS is inhibited

(Figure 1d). Note that in order to keep the intracellular concentration of MG-132 high, the drug efflux pump, PDR5, is also disrupted (62). We next determined whether Hrq1 is ubiquitylated by performing a ubiquitin pulldown experiment. We observe that Hrq1 is indeed ubiquitylated as observed as a smear and that Hrq1 ubiquitylation increases $30 \%$ following cisplatin exposure (Figure 1e; compare 6xHis-Ub pulldown lanes 2 and 4). These findings are strengthened by performing the reciprocal experiment where Hrq1 was pulled down and then blotted for ubiquitin (Supplemental Figure 1b). Together, these results demonstrate that Hrq1 is degraded by the proteasome following cisplatin treatment.

\section{The E3 ubiquitin ligase, Rad16, target Hrq1 for degradation following cisplatin.}

Since Hrq1 protein levels are regulated by the proteasome, we sought to identify the E3 ubiquitin ligase that target Hrq1. In S. cerevisiae, there are 60-100 E3 enzymes, so we prioritized E3 enzymes known to regulate DNA damage response proteins. For example, the NER gene, $\operatorname{Rad} 4$ (mammalian XPC), functions upstream of Hrq1 during crosslink repair, and its protein levels are regulated by the UPS following DNA damage $(21,63)$. Therefore, one possibility is that $\operatorname{Rad} 4$ and Hrq1 are targeted by the same E3 enzyme, Rad16 $(64,65)$. Thus, we examined whether Rad16 regulates Hrq1 protein levels. Indeed, we find that Hrq1 protein levels are largely stabilized in rad16 cells in both untreated and cisplatin treated conditions (Figure 2a). These results show that loss of $R A D 16 \mathrm{E} 3$ ubiquitin ligase results in Hrq1 protein stabilization following cisplatin exposure. 
We also examined potential E2 enzymes that regulate Hrq1. In yeast, there are only thirteen known E2s. Of the E2 enzymes only two, Ubc13 and Rad6, are associated with the DNA damage response (66). To test whether either of these two genes may regulate Hrq1, we knocked out either RAD6 or $U B C 13$ in a Hrq1-9myc tagged strain and performed cycloheximide chase experiments. Disruption of either $U B C 13$ or RAD6 only led to a mild stabilization of Hrq1 (Supplemental Figure 2). These results suggest there are likely additional E2s that regulate Hrq1 protein levels.

Hrq1 functions during error-free PRR. As stated above, DNA crosslinks are repaired using different pathways depending upon the cell cycle stage. For example, during replication, DNA intrastrand crosslinks are bypassed by the PRR pathway and then subsequently repaired by NER. Therefore, to determine whether Hrq1 functions during a specific cell cycle stage, we asked whether its protein levels are cell cycle regulated. We synchronized Hrq1-9xMYC expressing cells in G1 with alpha factor and released them into fresh medium to enable cell cycle progression. Protein extracts were made from equal cell numbers every 20 minutes and Hrq1 protein levels analyzed and compared to Kar2 as a loading control and Clb2 as a G2/M regulated cyclin. Consistent with a role during replication, Hrq1 protein levels begin to peak in S/G2 at 40 min after alpha factor release whereas $\mathrm{Clb} 2$ protein peaks starting at $60 \mathrm{~min}$ (Figure 3a). Cell cycle progression was confirmed by FACS analysis (Figure 3a). These results demonstrate that Hrq1 is enriched during S/G2, the cell cycle stage when PRR functions.

Previous studies in plants, fission yeast, and budding yeast have confounding results as to whether Hrq1 functions during PRR (44-46). Therefore, it remains controversial as to the role of 
Hrq1 during crosslink repair. To determine if Hrq1 has a role in PRR, we carefully and systematically examined the genetic relationship between $H R Q 1$ and multiple members of the PRR pathway. We combined HRQ1 mutants with disruption of PRR genes and tested these mutants for increased cisplatin sensitivity. Since Rad6/Rad18 ubiquitylates Pol30 during early PRR steps (Figure 3b), we began our analysis with rad6 $\Delta$ cells. When compared to wild-type, rad6 $\Delta$ cells are sensitive to $2.5 \mu \mathrm{g} / \mathrm{ml}$ cisplatin whereas $h r q 1 \Delta$ cells are sensitivity to $30 \mu \mathrm{g} / \mathrm{ml}$ cisplatin. Yeast disrupted with both $H R Q 1$ and $R A D 6$, exhibit sensitivity comparable to rad6 $\Delta$ alone (Figure 3c). These results suggest that $H R Q 1$ likely functions in the PRR pathway downstream of RAD6.

After Rad6/Rad18 mono-ubiquitinates PCNA, PCNA becomes poly-ubiquitinated on lysine 164 during error-free PRR (Figure 3b). At the same time, PCNA can be independently sumoylated on lysine 127, which recruits the DNA helicase Srs2 (not drawn). To address whether HRQ1 functions downstream of PCNA ubiquitylation, we examined the genetic relationship between HRQ1 and PCNA ubiquitylation/sumo mutants, POL30-K164R and POL30-K127R/K164R. Disruption of HRQ1 in combination with POL30-K164R or POL30-K127R/K164R, results in cisplatin sensitivity comparable to either POL30 single mutants (Figure 3d). These results are consistent with HRQ1 functioning downstream of POL30 ubiquitylation/sumoylation during PRR.

We next asked whether Hrq1 functions in the "error-free" template switching or "error-prone" translesion synthesis branches of PRR. To do this, we first examined the genetic relationship between HRQ1 and members of the "error-free" branch of PRR, RAD5-UBC13-MMS2, which 
polyubiquitylates PCNA (Figure 3b). Cells with both $H R Q 1$ and $R A D 5$ disrupted exhibit cisplatin sensitivity comparable to a $R A D 5$ single mutant (Figure 3e). These findings are consistent with a plant study demonstrating that RAD5 functions in the same pathway as $H R Q 1$, and contrast with a budding yeast study, where $H R Q 1$ was found to function independently of RAD5 (44-46). Suggesting that HRQ1 does indeed function during TS, we observe similar genetic results with another PRR member, $U B C 13$, where $h r q 1 \Delta u b c 13 \Delta$ double mutants exhibit cisplatin sensitivity to $u b c 13 \Delta$ (Figure 3e). Together, these results suggest that $H R Q 1$ functions in the "error-free" branch of PRR.

Next, we determined if HRQ1 also functions in the "error-prone" translesion synthesis branch of PRR by examining the genetic relationship between $H R Q 1$ and $R E V 1$, a translesion synthesis polymerase. We observe that $h r q 1 \Delta$ revld double mutant cells exhibit increased cisplatin sensitivity in comparison to either a $h r q 1 \Delta$ or a revl $\Delta$ single mutant (Figure 3f; $20 \mu \mathrm{g} / \mathrm{ml}$ cisplatin). These results suggest that Hrq1 functions primarily in the "error-free" branch of PRR.

\section{Hrq1 function and regulation in ICL repair is distinct from intrastrand crosslink repair.}

Loss of HRQ1 results in sensitivity to different types of DNA crosslinking agents including cisplatin and MMC. While both cisplatin and MMC cause ICLs and intrastrand crosslinks, cisplatin damage results in 90-95\% intrastrand crosslinks whereas mitomycin C results in 9095\% ICLs. Therefore by using MMC, we asked if Hrq1 has a similar function during ICL repair

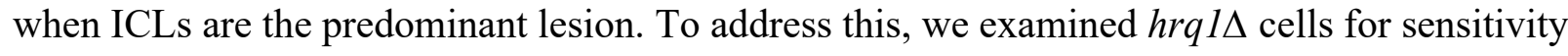
to MMC either alone or in combination with a rev1 $1 \Delta$ or $u b c 13 \Delta$ mutant (Supplemental Figure 3). As reported in a prior study (21), $h r q 1 \Delta$ cells are MMC sensitive (Supplemental Figure 3a). 
Like cisplatin induced DNA damage, the $h r q 1 \Delta$ revls double mutant exhibits increased MMC sensitive compared to the single mutants (Supplemental Figure 3a). These results suggest that $H R Q 1$ and $R E V 1$ are functioning in different pathways upon MMC exposure. However, in contrast to what we observed with cisplatin damage, a $h r q 1 \Delta u b c 13 \Delta$ double mutant exhibits increased MMC sensitivity compared to the single $h r q 1 \Delta$ or $u b c 13 \Delta$ mutants (Supplemental Figure 3a). These results suggest that Hrq1 function in ICL repair may be functionally distinct from intrastrand crosslink repair.

Since we observe genetic differences in $H R Q 1$ response to cisplatin- and MMC-induced DNA damage, we asked whether Hrq1 protein levels are similarly regulated upon MMC exposure by performing cycloheximide chase experiments (Supplemental Figure 3b). Quite surprisingly, and in contrast to the cisplatin-induced response, Hrq1 protein levels are largely stable following MMC exposure (compare Supplemental Figure 3b to Figure 1c). These results suggest that Hrq1 function and regulation in response to ICLs is indeed distinct from intrastrand crosslinks.

To validate Hrq1 role in repair of intrastrand crosslinks during PRR, we performed genetic analysis using UV-C, which induces primarily 6-4 photoproducts and cyclobutane pyrimidine dimers. Suggesting a role in template switch, we find that a hrq1 1 rad5 $\Delta$ double mutant has similar sensitivity to UV-C compared to $\operatorname{rad} 5 \Delta$. We observe similar results with $U B C 13$, as a hrq1 $u b c 13 \Delta$ double mutant has similar sensitivity to UV-C as a $u b c 13 \Delta$ single mutant (Supplemental Figure 3c). Suggesting $H R Q 1$ functions in a different pathway to TLS, a $h r q 1 \Delta$ rev $1 \Delta$ double mutant exhibits increased UV-C sensitive compared to the single mutants 
(Supplemental Figure 3d). Together these results strengthen the conclusion that Hrq1 functions during intrastrand crosslink repair via PRR.

Preventing Hrq1 degradation results in increased recombination and mutations. Since we find that Hrq1 functions during "error-free" PRR during intrastrand crosslink repair and is regulated by the $26 \mathrm{~S}$ proteasome, we sought to determine if misregulation of Hrq1 protein levels alter PRR. To do this, we determined the lysine residues that catalyze Hrq1 degradation upon cisplatin exposure (Figure 4a). Hrq1 is 1077 amino acids and contains 77 lysines. To identify potential ubiquitylation sites, we analyzed Hrq1 protein using UbPred, which predicts five ubiquitinated lysines (K164, K219, K221, K366, K872). In addition, we included two lysines that are conserved between Hrq1 and its mammalian ortholog, RECQL4 (K839, K938), as well as one lysine (K366), which is both predicted to be ubiquitylated by UbPred and conserved with RECQL4 $(67,68)$. We mutated these seven lysine residues to arginine at the endogenous HRQ1 locus and herein refer to this mutant as Hrq1-7KR. We next determined whether mutating these Hrq1 lysine residues results in Hrq1 protein stabilization. To test this, we performed cycloheximide chases on Hrq1-3xHA and Hrq1-7KR-6xHA. As previously observed, Hrq1 protein levels are reduced upon cisplatin treatment (Figures 1c, 4b). In contrast, Hrq1-7KR protein expression remains similar in both cisplatin treated and untreated conditions (Figure $\mathbf{4 b}$ ). It is interesting to note that the Hrq1-7KR mutant is not fully stabilized, suggesting that additional lysine residues may contribute to Hrq1 degradation independently of its DNA damage response function. These results suggest that the Hrq1-7KR mutant protein levels are misregulated in response to cisplatin. 
In Figure 1e, we show that Hrq1 is ubiquitylated and that its ubiquitylation increases $30 \%$ upon cisplatin exposure. Since Hrq1-7KR protein levels are stabilized upon cisplatin, we examined whether Hrq1-7KR ubiquiylation is altered. To address this, we performed ubiquitin pulldown experiments in the presence or absence of cisplatin as described in Figure 1e. Unlike wild-type Hrq1, we do not detect increased ubiquitylation of the Hrq1-7KR mutant upon cisplatin treatment (Figure 4c). To confirm these findings, we performed the reciprocal experiment where Hrq1-7KR was pulled down and then subsequently blotted for ubiquitin (Supplemental Figure 1C). However, we are unable to reliably detect ubiquitylated Hrq1-7KR either in the presence or absence of cisplatin (Supplemental Figure 1C). Together, these experiments suggest that mutation of these seven lysine residues results in deregulation of Hrq1 ubiquitylation upon cisplatin exposure resulting in its protein stabilization (Figure 4b,c).

We have thus far identified a function for Hrq1 in error-free PRR during intrastrand crosslink repair and found that Hrq1 is ubiquitylated and targeted for degradation upon cisplatin treatment. Therefore, we hypothesized that Hrq1 may need to be degraded for completion of PRR. Since Hrq1 functions during template switching, which is a recombination-based pathway, we asked whether stabilization of Hrq1 protein levels leads to increased recombination. To test this, we utilized the Hrq1-7KR mutant strain. At the same time, we created a strain where we induce Hrq1 over-expression (Hrq1 OE) by replacing HRQ1's endogenous promoter with a galactoseinducible/dextrose-repressible GAL1 promoter. GAL-3xHA-Hrq1 results in an approximately five-fold increase in Hrq1 expression compared to Hrq1-3xHA (Supplemental Figure 4a). Therefore, we used a Hrq1-7KR strain characterized above for our studies but treated the cells in 
the same manner as the GAL-3xHA-Hrq1 cells to enable direct comparisons between the two strains.

Using the Hrq1-7KR-6xHA mutant and the GAL-3xHA-Hrq1 strains, we performed a direct repeat recombination assay in the presence of galactose containing rich medium. We find that over-expression or stabilization of Hrq1 results in a 1.5 to 2 -fold increase in total recombination compared to WT cells following cisplatin treatment (Figure 4d). These results suggest high levels of Hrq1 following cisplatin exposure results in increased recombination.

Although recombination during template switching is considered "error-free", aberrant template switching can result in increased DNA mutations $(69,70)$. Therefore, we examined whether Hrq1 over-expression or stabilization increases mutagenesis. To measure mutation rates, we performed a canavanine mutagenesis assay. This assay measures mutations in the CAN1 permease gene, which enables cell viability upon exposure to the toxic arginine analog, canavanine. We observe over two-fold increase in mutation rates in Hrq1 over-expressing or 7KR cells in the presence cisplatin in comparison to WT cells (Figure 4e). These results suggest that Hrq1 must be tightly regulated to prevent excess recombination and mutagenesis.

Lastly, it is possible that preventing the degradation of Hrq1 results in accumulation of toxic DNA repair intermediates. To test this hypothesis, we examined the sensitivity of Hrq1-7KR cells upon cisplatin exposure (Supplemental Figure 4b). Surprisingly, we do not observe decreased cell viability of Hrq1-7KR cells compared to WT (Supplemental Figure 4b). This suggests that stabilization of Hrq1 during PRR may result in DNA repair intermediates that are 
ultimately resolved using an alternative pathway. Although we do not observe cisplatin sensitivity in the Hrq1-KR mutant, overexpression of the HRQ1-7KR (by using a galactose inducible promoter) results in cell lethality even in the absence of DNA damage (Supplemental Figure 4c).

\section{Similar to Hrq1, the levels of RECQL4 decreases following treatment to DNA crosslinking}

agents. We next sought to determine whether there is a conserved regulatory role for human RECQL4 following cisplatin exposure. We used U2OS cells since they are commonly used in DNA repair studies and Rothmund-Thompson patients with RECQL4 mutations are predisposed to osteosarcomas. Since RECQL4 is needed for cisplatin resistance, we hypothesized that similar to Hrq1 its protein levels may also decrease follow cisplatin exposure. To test this hypothesis, we repeated our cycloheximide chase experiments in the U2OS cell line. Similar to yeast Hrq1, RECQL4 protein levels decrease following cisplatin exposure in comparison to the untreated control (Figure 5a).

We next determined whether this phenomenon holds true with an endogenous crosslinking agent, acetaldehyde. Acetaldehyde is known as the toxic byproduct of alcohol metabolism, but it is also naturally produced from the breakdown of various foods including yogurt, apples, etc. $(71,72)$. Acetaldehyde is highly reactive and can produce similar lesions to cisplatin $(73,74)$. Like cisplatin, RECQL4 levels decrease following acetaldehyde exposure (Figure 5b). Suggesting that RECQL4 degradation is also lesion specific, a prior study showed that RECQL4 protein levels are maintained following IR or camptothecin exposure (75). Together, these results 
suggest that there is a conserved regulatory mechanism between yeast Hrq1 and human RECQL4 during DNA crosslink repair.

Since we find that Hrq1 functions in the error-free branch of PRR, we hypothesized that RECQL4 functions similarly. To test this, we determined the cisplatin sensitivity of U2OS cells with siRNA knockdown of RECQL4 alone or in combination with the error-prone PRR polymerase, $R E V 1$. Using an MTS assay, we find that both siRECQL4 and siREV1 individually treated cells are cisplatin sensitive (Figure 5c). If RECQL4 and REVI function in the same PRR branch, we expect that siRNA knockdown of both RECQL4 and REVI will result in similar cisplatin sensitivity as the single knockdowns. However, if RECQL4 and REV1 function in different pathways, we expect increased cisplatin sensitivity when both RECQL4 and REVI are knocked down. Consistent with RECQL4 and REV1 functioning in different pathways, we observe increased cisplatin sensitivity in the double treated siRECQL4 siREV1 cells using both MTS and clonogenic survival assays (Figure 5 c,d, respectively). These results suggest that, like $H R Q 1, R E C Q L 4$ may function in the error-free PRR pathway.

Since yeast Hrq1 over-expression led to increased recombination, we examined whether overexpression of human RECQL4 also leads to increased recombination. To do this, we created a plasmid that over-expressed RECQL4 (Supplemental Figure 4d) and measured these cells for RAD51 focus formation in the presence or absence of cisplatin. RAD51 is a central homologous recombination protein that is relocated to DNA repair sites following DNA damage. Suggesting that there is increased recombination, we find that RECQL4 over-expression results in a significant increase in the number of cells with RAD51 foci in both untreated and cisplatin 
treated cells (Figure 6a; $\mathrm{p}<0.0001$ for both). Since RECQL4 is a DNA helicase, we asked whether its helicase activity would be crucial for RAD51 focus formation upon cisplatin exposure. Suggesting that RECQL4 DNA unwinding activity likely contributes to RAD51 focus formation, we observe that that RECQL4 helicase mutant, RECQL4-K508A, exhibits reduced RAD51 foci compared to RECQL4 over-expressing cells (Figure 6a; $p<0.01$ ). Therefore, the RECQL4 protein family has a conserved function in mediating recombination during DNA crosslink repair.

The RAD51 foci observed upon RECQL4 over-expression could be indicative of increased DSB formation, recombination, or even ssDNA from excess RECQL4-mediated DNA unwinding. To examine whether RECQL4 overexpression leads to increased DSB formation, we performed neutral comet assays. We find that RECQL4 over-expression in untreated or cisplatin-treated cells do not increase tail moments compared to the empty control U2OS cell line (Figure 6b). Intriguingly, RECQL4 over-expression decreases tail moments upon cisplatin exposure. Suggesting that RECQL4 helicase activity is critical to prevent DSB formation following cisplatin, the RECQL4 helicase mutant, RECQL4-K508A, has similar tail moments to the empty control (Figure 6b). These finding are consistent with a role for RECQL4 in preventing DSB formation and fork collapse, perhaps, by enabling lesion bypass through TS.

It is possible is that the increased RAD51 foci observed may be due to more ssDNA caused by RECQL4 DNA unwinding. To examine whether RECQL4 over-expression results in more ssDNA, we quantitated the tail moments following S1 nuclease treatment. If more ssDNA is present, the S1 nuclease will degrade the ssDNA resulting in more DSBs. Although statistically 
insignificant, we find that RECQL4 over-expression extremely modestly increases ssDNA and that this increase is helicase dependent (Figure 6b). Overall, our suggests that RECQL4 overexpression does not significantly increase either DSBs or ssDNA. Therefore, the increased RAD51 foci observed upon RECQL4-over expression could be due to increased recombination happening at the replication fork.

\section{Bioinformatic analysis reveals elevated RECQL4 levels are associated with tumorigenesis}

and increased tumor mutation burden. We find that Hrq1/RECQL4 over-expression results in genomic instability, which is a cancer hallmark. Therefore, we asked whether RECQL4 is overexpressed in breast tumors relative to normal tissue. By examining mRNA levels of RECQL4 in TCGA, we observe that RECQL4 expression is increased in tumors versus normal tissues (Figure 7a). Several tumor types where RECQL4 is over-expressed are treated with cisplatin, including breast cancer. Therefore, we focused on breast cancer where we can delineate between breast cancers that are classically treated with cisplatin versus those that are not. Estrogen receptor negative (ER-) breast cancers are generally more aggressive and typically treated with cisplatin including TNBC, an ER- breast cancer subtype (76). In contrast, ER+ breast cancers are classically treated with hormone therapy (76). When compared to ER+ breast cancers, ER- breast cancers have elevated levels of RECQL4 expression (Figure 7b). Our result is consistent with another study where RECQL4 expression was elevated in more aggressive cancers (14).

As elevated Hrq1 protein levels in yeast result in increased mutations, we postulated that increased human RECQL4 expression correlates with increased tumor mutation burden. Indeed, 
when examining ER- cancers, high levels of RECQL4 correlates with increased mutational burden (Figure 7c).

When yeast Hrq1 is over-expressed, we find that both mutations and recombination are increased. Therefore, we asked whether over-expression of RECQL4 results in increased expression of recombination genes. Using the METABRIC and TCGA mRNA expression datasets, we examined which cellular pathways are enriched in RECQL4 over-expressing ERtumors. This analysis revealed that cell cycle progression, transcriptional regulation, and DNA repair are enriched in RECQL4 high subset of TNBC (Figure 7d). Upon further analysis, we find that high levels of RECQL4 significantly correlates with genes in the HR pathway and specifically with RAD51 (Figure 7e). These results are consistent with our yeast and mammalian studies here suggesting that high levels of RECQL4 correlate with increased mutations and recombination.

In tumor cells, DNA repair defects can result in therapeutic sensitivity. Since RECQL4 overexpression leads to increased genomic instability, we asked whether RECQL4 expression may predict therapeutic response to cisplatin. In cisplatin-treated TNBCs, tumors with elevated RECQL4 expression have a better clinical response compared to tumors with lower RECQL4 expression (Figure 7f,g). This suggests that increased RECQL4 levels may provide a prognostic marker for therapeutic response to cisplatin.

\section{Discussion}


DNA crosslinks can arise endogenously from reactive aldehydes, or exogenously from chemotherapeutic agents such as cisplatin. DNA crosslink repair is critical to maintain genomic stability and for disease prevention. While the RECQL4 family has a conserved role in DNA crosslink repair, its role during this process is poorly understood. Here we use the yeast model to identify a function for the RECQL4 family in DNA crosslink repair by placing yeast Hrq1 in the template switching pathway to mediate bypass of intrastrand crosslinks. Furthermore, we find that Hrq1 is regulated by the UPS during instrastrand crosslink repair and that misregulation of Hrq1 protein levels results in increased recombination and mutagenesis. We extend this analysis to mammalian cells and show that like yeast, human RECQL4 protein levels are depleted in response to DNA crosslinks. Using bioinformatic/meta-analysis, we show that increased RECQL4 levels is associated with increased tumor burden, mutations, and therapeutic response. Together our studies demonstrate a conserved role for the RECQL4 protein family in crosslink repair whose levels must be fine-tuned to prevent excess recombination and mutations.

Our results show that Hrq1/RECQL4 protein levels are tightly regulated to ensure accurate repair of DNA intrastrand crosslinks. Protein degradation plays a crucial regulatory role during DNA repair. While both Hrq1 and RECQL4 are needed for resistance to crosslinking agents, their protein levels decrease upon exposure to cisplatin or acetaldehyde (Figures 1 and 5). Consistent with this observation, proteasome-mediated degradation of other DNA helicases (BLM, FBH1, HLTF, amongst others) was observed following DNA damage (77). Degradation of these key helicases is critical for dictating which pathway is used as well as preventing accumulation of toxic DNA repair intermediates. Thus, degradation of DNA helicases serves an important role in maintaining genome homeostasis. A prior study suggested that RECQL4 is not targeted by the 
ubiquitin proteasome in HeLa cells even though it directly interacts with the E3 ubiquitin ligases, UBR1 and UBR2 (78). However, this study was not performed with DNA damage. Therefore, it is possible that RECQL4 may be ubiquitylated in a DNA damage-dependent manner or that there could be cell type specific differences. Consistent with this notion, recent studies demonstrate that RECQL4 ubiquitylation plays a critical role in double-strand break repair $(79,80)$. However, RECQL4 protein levels were not assessed in these studies $(79,80)$. Another study analyzed RECQL4 protein level following IR or camptothecin treatment and found that it was unchanged; however, other DNA damaging agents were not tested (75). It is important to note that in budding yeast, Hrq1 proteasomal degradation is specific for DNA intrastrand crosslinks (Figure 1b,

\section{Supplemental Figure 3b).}

Functional analysis of the RECQL4 protein family during crosslink repair is best described for ICLs in yeast. After a DNA intrastrand crosslink occurs during DNA replication, the crosslink can result in fork stalling which leads to mono-ubiquitylation of PCNA by Rad6/Rad18 (Figure

7). Subsequently, the lesion is bypassed by the translesion polymerase, Rev1, or alternatively, polyubiquitylation by Rad5-Ubc13-Mms2 promotes a template switching mechanism. Our model shows that Hrq1 mediates template switching and is subsequently ubiquitylated by Rad16 and degraded by the proteasome (Figure 7). After the replication fork passes, then NER mediates removal of the intrastrand crosslink. This mechanism is likely conserved in humans as RECQL4 knockdown results in cisplatin sensitivity and altered replication fork dynamics (Arora et al. 2016). Suggesting a conserved function, biochemical analysis demonstrates that both Hrq1 and RECQL4 bind and unwind similar DNA substrates including bubbles, D-loops, and poly(dT) forks (22). Our studies here demonstrate that like RECQL4, Hrq1 also plays a key role during 
replication and is cell cycle regulated, peaking in the S/G2 cell cycle phase. Together our results suggest a conserved mechanism for the RECQL4 family during replication-associated intrastrand crosslink repair (Figure 7).

Previous studies presented conflicting results as to whether Hrq1 functions during PRR (44-46). There are multiple explanations for this discrepancy. For example, in $S$. pombe polyubiquitylation of PCNA (Pol30) triggers both TS and TLS. Therefore, the synthetic lethality observed between fission yeast $H R Q 1$ and factors that mediate PCNA polyubiquitylation may be due to function in TLS rather than an independent function in TS (44). Separation-of-function alleles are needed to differentiate between these two functions. Our study also contradicts another in S. cerevisiae, which found that Hrq1 functions independently of PRR (45). It is possible that there are background strain differences since we used W303 and the other group used the YPH499 genetic background (derived from YNN216). It is important to note that our study here performed extensive genetic analysis at each step of the PRR pathway whereas the other study only analyzed RAD5 and REV3 mutants (45). Furthermore, we find that our results are consistent with UV-C damage as well (Supplemental Figure 3c). Lastly, we note that our results are consistent with those from plants where Hrq1 was found to function in PRR (46) and with our genetic analysis in mammalian cells (Figure 5). Altogether, we provide strong evidence that Hrq1 promotes recombination during TS to mediate repair of intrastrand crosslinks.

\section{Data Availability}

The data supporting the findings of this study are available within the article and/or its supplementary materials. 


\section{ACKNOWLEDGEMENTS}

This study was supported by grants from the National Institutes of Health (ES030335 to K.A.B.) and the Department of Defense (BC201356 to K.A.B.). This work was also supported by the Hillman Fellows for Innovative Cancer Research Program to K.A.B.

\section{Author Contributions}

T.T.L. and K.A.B. conceptualized the study and wrote the manuscript. T.T.L., Z.L., N.P., P.S.P. S.B., K.R., A.V.L., and K.A.B. were involved in data acquisition/analysis and figure creations. 


\section{Bibliography}

1. Hickson,I.D. (2003) RecQ helicases: caretakers of the genome. Nat. Rev. Cancer, 3, 169-178.

2. Bohr,V.A. (2008) Rising from the RecQ-age: the role of human RecQ helicases in genome maintenance. Trends Biochem. Sci., 33, 609-620.

3. Chu,W.K. and Hickson,I.D. (2009) RecQ helicases: multifunctional genome caretakers. Nat. Rev. Cancer, 9, 644-654.

4. Bernstein,K.A., Gangloff,S. and Rothstein,R. (2010) The RecQ DNA helicases in DNA repair. Annu. Rev. Genet., 44, 393-417.

5. Croteau,D.L., Popuri,V., Opresko,P.L. and Bohr,V.A. (2014) Human RecQ helicases in DNA repair, recombination, and replication. Annu. Rev. Biochem., 83, 519-552.

6. Kääriäinen,H., Ryöppy,S. and Norio,R. (1989) RAPADILINO syndrome with radial and patellar aplasia/hypoplasia as main manifestations. Am. J. Med. Genet., 33, 346-351.

7. Kitao,S., Shimamoto,A., Goto,M., Miller,R.W., Smithson,W.A., Lindor,N.M. and Furuichi,Y. (1999) Mutations in RECQL4 cause a subset of cases of Rothmund-Thomson syndrome. Nat. Genet., 22, 82-84.

8. Hoki,Y., Araki,R., Fujimori,A., Ohhata,T., Koseki,H., Fukumura,R., Nakamura,M., Takahashi,H., Noda,Y., Kito,S., et al. (2003) Growth retardation and skin abnormalities of the Recql4-deficient mouse. Hum. Mol. Genet., 12, 2293-2299.

9. Siitonen,H.A., Kopra,O., Kääriäinen,H., Haravuori,H., Winter,R.M., Säämänen,A.-M., Peltonen,L. and Kestilä,M. (2003) Molecular defect of RAPADILINO syndrome expands the phenotype spectrum of RECQL diseases. Hum. Mol. Genet., 12, 2837-2844.

10. Larizza,L., Magnani,I. and Roversi,G. (2006) Rothmund-Thomson syndrome and RECQL4 defect: splitting and lumping. Cancer Lett., 232, 107-120.

11. Van Maldergem,L., Siitonen,H.A., Jalkh,N., Chouery,E., De Roy,M., Delague,V., Muenke,M., Jabs,E.W., Cai,J., Wang,L.L., et al. (2006) Revisiting the craniosynostosisradial ray hypoplasia association: Baller-Gerold syndrome caused by mutations in the RECQL4 gene. J. Med. Genet., 43, 148-152.

12. Larizza,L., Roversi,G. and Volpi,L. (2010) Rothmund-Thomson syndrome. Orphanet J Rare Dis, $\mathbf{5}, 2$.

13. Su,Y., Meador,J.A., Calaf,G.M., Proietti De-Santis,L., Zhao,Y., Bohr,V.A. and Balajee,A.S. (2010) Human RecQL4 helicase plays critical roles in prostate carcinogenesis. Cancer Res., 70, 9207-9217.

14. Arora,A., Agarwal,D., Abdel-Fatah,T.M., Lu,H., Croteau,D.L., Moseley,P., Aleskandarany,M.A., Green,A.R., Ball,G., Rakha,E.A., et al. (2016) RECQL4 helicase has oncogenic potential in sporadic breast cancers. J. Pathol., 238, 495-501. 
15. Mo,D., Fang,H., Niu,K., Liu,J., Wu,M., Li,S., Zhu,T., Aleskandarany,M.A., Arora,A., Lobo,D.N., et al. (2016) Human Helicase RECQL4 Drives Cisplatin Resistance in Gastric Cancer by Activating an AKT-YB1-MDR1 Signaling Pathway. Cancer Res., 76, 3057-3066.

16. Li,J., Jin,J., Liao,M., Dang,W., Chen,X., Wu,Y. and Liao,W. (2018) Upregulation of RECQL4 expression predicts poor prognosis in hepatocellular carcinoma. Oncol. Lett., $15,4248-4254$.

17. Ichikawa,K., Noda,T. and Furuichi,Y. (2002) [Preparation of the gene targeted knockout mice for human premature aging diseases, Werner syndrome, and Rothmund-Thomson syndrome caused by the mutation of DNA helicases]. Nihon Shinkei Seishin Yakurigaku Zasshi.

18. Smeets,M.F., DeLuca,E., Wall,M., Quach,J.M., Chalk,A.M., Deans,A.J., Heierhorst,J., Purton,L.E., Izon,D.J. and Walkley,C.R. (2014) The Rothmund-Thomson syndrome helicase RECQL4 is essential for hematopoiesis. J. Clin. Invest., 124, 3551-3565.

19. Fang,H., Niu,K., Mo,D., Zhu,Y., Tan,Q., Wei,D., Li,Y., Chen,Z., Yang,S., Balajee,A.S., et al. (2018) RecQL4-Aurora B kinase axis is essential for cellular proliferation, cell cycle progression, and mitotic integrity. Oncogenesis, 7, 68.

20. Barea,F., Tessaro,S. and Bonatto,D. (2008) In silico analyses of a new group of fungal and plant RecQ4-homologous proteins. Comput Biol Chem, 32, 349-358.

21. Bochman,M.L., Paeschke,K., Chan,A. and Zakian,V.A. (2014) Hrq1, a homolog of the human RecQ4 helicase, acts catalytically and structurally to promote genome integrity. Cell Rep., 6, 346-356.

22. Rogers,C.M., Wang,J.C.-Y., Noguchi,H., Imasaki,T., Takagi,Y. and Bochman,M.L. (2017) Yeast Hrq1 shares structural and functional homology with the disease-linked human RecQ4 helicase. Nucleic Acids Res., 45, 5217-5230.

23. O’Donovan,A., Davies,A.A., Moggs,J.G., West,S.C. and Wood,R.D. (1994) XPG endonuclease makes the 3 ' incision in human DNA nucleotide excision repair. Nature, 371, 432-435.

24. Bauer,G.B. and Povirk,L.F. (1997) Specificity and kinetics of interstrand and intrastrand bifunctional alkylation by nitrogen mustards at a G-G-C sequence. Nucleic Acids Res., 25, 1211-1218.

25. Deans,A.J. and West,S.C. (2011) DNA interstrand crosslink repair and cancer. Nat. Rev. Cancer, 11, 467-480.

26. Lopez-Martinez,D., Liang,C.-C. and Cohn,M.A. (2016) Cellular response to DNA interstrand crosslinks: the Fanconi anemia pathway. Cell Mol. Life Sci., 73, 3097-3114.

27. Moldovan,G.-L. and D'Andrea,A.D. (2009) How the fanconi anemia pathway guards the genome. Annu. Rev. Genet., 43, 223-249. 
28. McHugh,P.J., Ward,T.A. and Chovanec,M. (2012) A prototypical Fanconi anemia pathway in lower eukaryotes? Cell Cycle, 11, 3739-3744.

29. Ward,T.A., Dudášová,Z., Sarkar,S., Bhide,M.R., Vlasáková,D., Chovanec,M. and McHugh,P.J. (2012) Components of a Fanconi-like pathway control Pso2-independent DNA interstrand crosslink repair in yeast. PLoS Genet., 8, e1002884.

30. Kottemann,M.C. and Smogorzewska,A. (2013) Fanconi anaemia and the repair of Watson and Crick DNA crosslinks. Nature, 493, 356-363.

31. Ceccaldi,R., Sarangi,P. and D’Andrea,A.D. (2016) The Fanconi anaemia pathway: new players and new functions. Nat. Rev. Mol. Cell Biol., 17, 337-349.

32. McHugh,P.J. and Sarkar,S. (2006) DNA interstrand cross-link repair in the cell cycle: a critical role for polymerase zeta in G1 phase. Cell Cycle, 5, 1044-1047.

33. Noll,D.M., Mason,T.M. and Miller,P.S. (2006) Formation and repair of interstrand crosslinks in DNA. Chem. Rev., 106, 277-301.

34. Shen,X. and Li,L. (2010) Mutagenic repair of DNA interstrand crosslinks. Environ Mol Mutagen, 51, 493-499.

35. Daee,D.L. and Myung,K. (2012) Fanconi-like crosslink repair in yeast. Genome Integr, 3, 7.

36. Budzowska,M. and Kanaar,R. (2009) Mechanisms of dealing with DNA damage-induced replication problems. Cell Biochem. Biophys., 53, 17-31.

37. Ulrich,H.D. and Walden,H. (2010) Ubiquitin signalling in DNA replication and repair. Nat. Rev. Mol. Cell Biol., 11, 479-489.

38. Köberle,B., Masters,J.R., Hartley,J.A. and Wood,R.D. (1999) Defective repair of cisplatininduced DNA damage caused by reduced XPA protein in testicular germ cell tumours. Curr. Biol., 9, 273-276.

39. McKay,B.C., Becerril,C. and Ljungman,M. (2001) P53 plays a protective role against UVand cisplatin-induced apoptosis in transcription-coupled repair proficient fibroblasts. Oncogene, 20, 6805-6808.

40. Welsh,C., Day,R., McGurk,C., Masters,J.R.W., Wood,R.D. and Köberle,B. (2004) Reduced levels of XPA, ERCC1 and XPF DNA repair proteins in testis tumor cell lines. Int. J. Cancer, 110, 352-361.

41. Basu,A. and Krishnamurthy,S. (2010) Cellular responses to Cisplatin-induced DNA damage. J. Nucleic Acids, 2010.

42. Branzei,D. and Foiani,M. (2010) Maintaining genome stability at the replication fork. Nat. Rev. Mol. Cell Biol., 11, 208-219.

43. Berti,M., Cortez,D. and Lopes,M. (2020) The plasticity of DNA replication forks in response to clinically relevant genotoxic stress. Nat. Rev. Mol. Cell Biol., 21, 633-651. 
44. Groocock,L.M., Prudden,J., Perry,J.J.P. and Boddy,M.N. (2012) The RecQ4 orthologue Hrq1 is critical for DNA interstrand cross-link repair and genome stability in fission yeast. Mol. Cell. Biol., 32, 276-287.

45. Choi,D.-H., Min,M.-H., Kim,M.-J., Lee,R., Kwon,S.-H. and Bae,S.-H. (2014) Hrq1 facilitates nucleotide excision repair of DNA damage induced by 4-nitroquinoline-1oxide and cisplatin in Saccharomyces cerevisiae. J. Microbiol., 52, 292-298.

46. Röhrig,S., Dorn,A., Enderle,J., Schindele,A., Herrmann,N.J., Knoll,A. and Puchta,H. (2018) The RecQ-like helicase HRQ1 is involved in DNA crosslink repair in Arabidopsis in a common pathway with the Fanconi anemia-associated nuclease FAN1 and the postreplicative repair ATPase RAD5A. New Phytol., 218, 1478-1490.

47. Gupta,S.V. and Schmidt,K.H. (2020) Maintenance of yeast genome integrity by recq family DNA helicases. Genes (Basel), 11.

48. Thomas,B.J. and Rothstein,R. (1989) Elevated recombination rates in transcriptionally active DNA. Cell, 56, 619-630.

49. Zhao,X., Muller,E.G. and Rothstein,R. (1998) A suppressor of two essential checkpoint genes identifies a novel protein that negatively affects dNTP pools. Mol. Cell, 2, 329340 .

50. Sherman,F. (2002) Getting started with yeast. Meth. Enzymol., 350, 3-41.

51. Janke,C., Magiera,M.M., Rathfelder,N., Taxis,C., Reber,S., Maekawa,H., MorenoBorchart,A., Doenges,G., Schwob,E., Schiebel,E., et al. (2004) A versatile toolbox for PCR-based tagging of yeast genes: new fluorescent proteins, more markers and promoter substitution cassettes. Yeast, 21, 947-962.

52. Böhm,S., Mihalevic,M.J., Casal,M.A. and Bernstein,K.A. (2015) Disruption of SUMOtargeted ubiquitin ligases S1x5-Slx8/RNF4 alters RecQ-like helicase Sgs1/BLM localization in yeast and human cells. DNA Repair (Amst.), 26, 1-14.

53. Knop,M., Siegers,K., Pereira,G., Zachariae,W., Winsor,B., Nasmyth,K. and Schiebel,E. (1999) Epitope tagging of yeast genes using a PCR-based strategy: more tags and improved practical routines.

54. Böhm,S., Szakal,B., Herken,B.W., Sullivan,M.R., Mihalevic,M.J., Kabbinavar,F.F., Branzei,D., Clark,N.L. and Bernstein,K.A. (2016) The budding yeast ubiquitin protease ubp7 is a novel component involved in S phase progression. J. Biol. Chem., 291, 44424452.

55. Muratani,M., Kung,C., Shokat,K.M. and Tansey,W.P. (2005) The F box protein Dsg1/Mdm30 is a transcriptional coactivator that stimulates Gal4 turnover and cotranscriptional mRNA processing. Cell, 120, 887-899.

56. Tansey,W.P. (2006) Detection of ubiquitylated proteins in yeast. CSH Protoc, 2006. 
57. Godin,S.K., Zhang,Z., Herken,B.W., Westmoreland,J.W., Lee,A.G., Mihalevic,M.J., Yu,Z., Sobol,R.W., Resnick,M.A. and Bernstein,K.A. (2016) The Shu complex promotes errorfree tolerance of alkylation-induced base excision repair products. Nucleic Acids Res., 44, 8199-8215.

58. Geissmann,Q. (2013) OpenCFU, a new free and open-source software to count cell colonies and other circular objects. PLoS One, 8, e54072.

59. Godin,S., Wier,A., Kabbinavar,F., Bratton-Palmer,D.S., Ghodke,H., Van Houten,B., VanDemark,A.P. and Bernstein,K.A. (2013) The Shu complex interacts with Rad51 through the Rad51 paralogues Rad55-Rad57 to mediate error-free recombination. Nucleic Acids Res., 41, 4525-4534.

60. Guzmán,C., Bagga,M., Kaur,A., Westermarck,J. and Abankwa,D. (2014) ColonyArea: an ImageJ plugin to automatically quantify colony formation in clonogenic assays. PLoS One, 9, e92444.

61. Vlachostergios,P.J., Patrikidou,A., Daliani,D.D. and Papandreou,C.N. (2009) The ubiquitinproteasome system in cancer, a major player in DNA repair. Part 1: post-translational regulation. J. Cell Mol. Med., 13, 3006-3018.

62. Tran,J.R. and Brodsky,J.L. (2012) Assays to measure ER-associated degradation in yeast. Methods Mol. Biol., 832, 505-518.

63. Lommel,L., Ortolan,T., Chen,L., Madura,K. and Sweder,K.S. (2002) Proteolysis of a nucleotide excision repair protein by the $26 \mathrm{~S}$ proteasome. Curr. Genet., 42, 9-20.

64. Ramsey,K.L., Smith,J.J., Dasgupta,A., Maqani,N., Grant,P. and Auble,D.T. (2004) The NEF4 complex regulates Rad4 levels and utilizes Snf2/Swi2-related ATPase activity for nucleotide excision repair. Mol. Cell. Biol., 24, 6362-6378.

65. Gillette,T.G., Yu,S., Zhou,Z., Waters,R., Johnston,S.A. and Reed,S.H. (2006) Distinct functions of the ubiquitin-proteasome pathway influence nucleotide excision repair. EMBO J., 25, 2529-2538.

66. Finley,D., Ulrich,H.D., Sommer,T. and Kaiser,P. (2012) The ubiquitin-proteasome system of Saccharomyces cerevisiae. Genetics, 192, 319-360.

67. Radivojac,P., Vacic,V., Haynes,C., Cocklin,R.R., Mohan,A., Heyen,J.W., Goebl,M.G. and Iakoucheva,L.M. (2010) Identification, analysis, and prediction of protein ubiquitination sites. Proteins, 78, 365-380.

68. Madeira,F., Park,Y.M., Lee,J., Buso,N., Gur,T., Madhusoodanan,N., Basutkar,P., Tivey,A.R.N., Potter,S.C., Finn,R.D., et al. (2019) The EMBL-EBI search and sequence analysis tools APIs in 2019. Nucleic Acids Res., 47, W636-W641.

69. Lovett,S.T. (2017) Template-switching during replication fork repair in bacteria. DNA Repair (Amst.), 56, 118-128. 
70. Löytynoja,A. and Goldman,N. (2017) Short template switch events explain mutation clusters in the human genome. Genome Res., 27, 1039-1049.

71. Langevin,F., Crossan,G.P., Rosado,I.V., Arends,M.J. and Patel,K.J. (2011) Fancd2 counteracts the toxic effects of naturally produced aldehydes in mice. Nature, 475, 5358 .

72. Uebelacker,M. and Lachenmeier,D.W. (2011) Quantitative determination of acetaldehyde in foods using automated digestion with simulated gastric fluid followed by headspace gas chromatography. J. Autom. Methods Manag. Chem., 2011, 907317.

73. Schärer,O.D. (2005) DNA interstrand crosslinks: natural and drug-induced DNA adducts that induce unique cellular responses. Chembiochem, 6, 27-32.

74. Sonohara,Y., Yamamoto,J., Tohashi,K., Takatsuka,R., Matsuda,T., Iwai,S. and Kuraoka,I. (2019) Acetaldehyde forms covalent GG intrastrand crosslinks in DNA. Sci. Rep., 9, 660.

75. Shamanna,R.A., Lu,H., Croteau,D.L., Arora,A., Agarwal,D., Ball,G., Aleskandarany,M.A., Ellis,I.O., Pommier,Y., Madhusudan,S., et al. (2016) Camptothecin targets WRN protein: mechanism and relevance in clinical breast cancer. Oncotarget, 7, 13269-13284.

76. Sheikh,M.S., Shao,Z.M., Li,X.S., Dawson,M., Jetten,A.M., Wu,S., Conley,B.A., Garcia,M., Rochefort,H. and Fontana,J.A. (1994) Retinoid-resistant estrogen receptor-negative human breast carcinoma cells transfected with retinoic acid receptor-alpha acquire sensitivity to growth inhibition by retinoids. J. Biol. Chem., 269, 21440-21447.

77. Sommers,J.A., Suhasini,A.N. and Brosh,R.M. (2015) Protein degradation pathways regulate the functions of helicases in the DNA damage response and maintenance of genomic stability. Biomolecules, 5, 590-616.

78. Yin,J., Kwon,Y.T., Varshavsky,A. and Wang,W. (2004) RECQL4, mutated in the Rothmund-Thomson and RAPADILINO syndromes, interacts with ubiquitin ligases UBR1 and UBR2 of the N-end rule pathway. Hum. Mol. Genet., 13, 2421-2430.

79. Lu,H., Shamanna,R.A., de Freitas,J.K., Okur,M., Khadka,P., Kulikowicz,T., Holland,P.P., Tian,J., Croteau,D.L., Davis,A.J., et al. (2017) Cell cycle-dependent phosphorylation regulates RECQL4 pathway choice and ubiquitination in DNA double-strand break repair. Nat. Commun., 8, 2039.

80. Tan,Q., Niu,K., Zhu,Y., Chen,Z., Li,Y., Li,M., Wei,D., Balajee,A.S., Fang,H. and Zhao,Y. (2021) RNF8 ubiquitinates RecQL4 and promotes its dissociation from DNA double strand breaks. Oncogenesis, 10, 24. 


\section{Figure 1}

a.

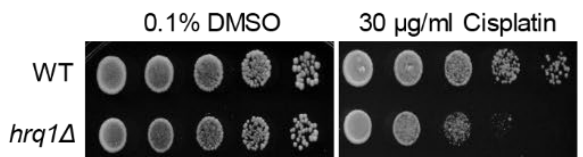

b.

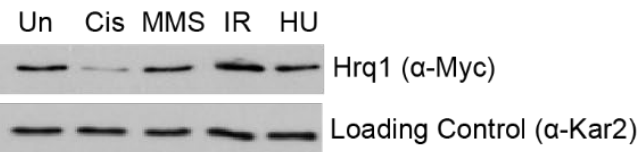

C.
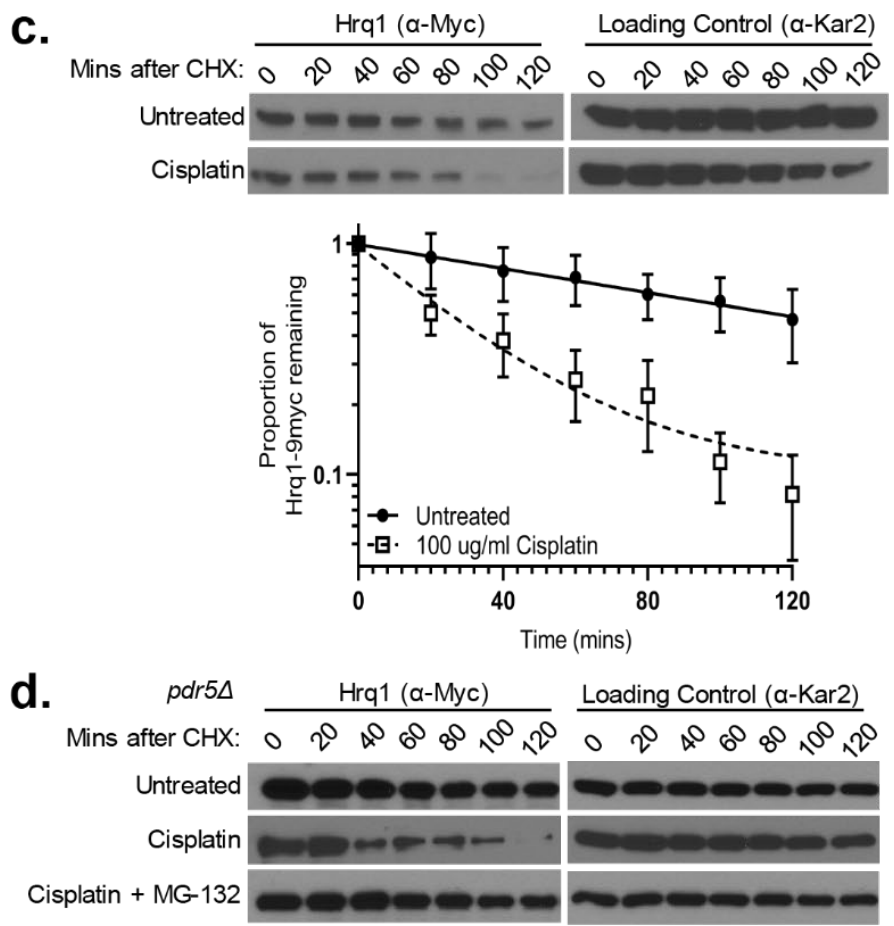

e.
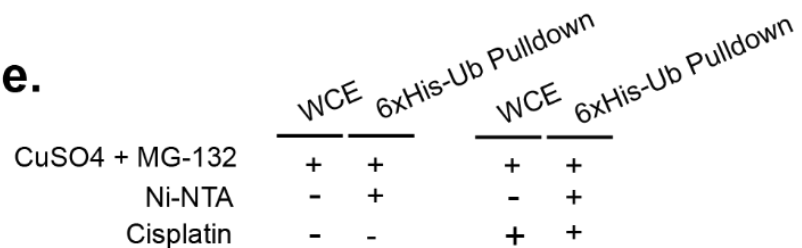

Hrq1 (a-Myc)

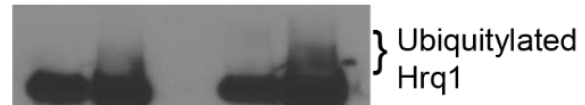

Pulldown/WCE

1

1.3

\section{Figure Legends}

Figure 1. Although Hrq1 is needed for cisplatin resistance, it is degraded by the proteasome

upon cisplatin exposure. a) $H R Q 1$-null cells are sensitive to cisplatin. Wild-type (WT) or $h r q 1 \Delta$ 
disrupted cells were five-fold serially diluted on medium containing $30 \mu \mathrm{g} / \mathrm{ml}$ cisplatin and/or $0.1 \%$ DMSO, grown for 48 hours at $30^{\circ} \mathrm{C}$, and photographed. b) Hrq1 level is stable following treatment with other DNA damaging agents. Exponentially growing cells with Hrq1-9xMYC were treated with the indicated drugs for 2 hours before being harvested for western. c) Hrq1 protein levels are decreased upon cisplatin treatment. Exponentially growing cells with Hrq19xMYC were incubated with cycloheximide in the presence or absence of $100 \mu \mathrm{g} / \mathrm{ml}$ cisplatin and/or $0.1 \%$ DMSO. Quantification of the proportion of Hrq1 remaining relative to time 0 (before CHX addition) and the loading control, Kar2. The experiment was performed five times with mean and standard error plotted. d) The proteasome degrades Hrq1 following cisplatin exposure. PDR5 disrupted cells were untreated (0.1\% DMSO), cisplatin treated, or pretreated for one hour with $50 \mu \mathrm{M}$ MG-132 before cisplatin addition with $0.1 \%$ DMSO. Cycloheximide chases were performed the same as b). e) Hrq1 is ubiquitylated in vivo. Lanes 1 (whole cell extract, WCE without pulldown, 10\% input) serves as the control whereas lane 2 is the pulldown showing ubiquitylated Hrq1 as indicated. Lanes 3 and 4 is after treatment with cisplatin, where lane 3 is WCE and lane 4 is pulldown showing increased ubiquitylated Hrq1. 


\section{Figure 2}

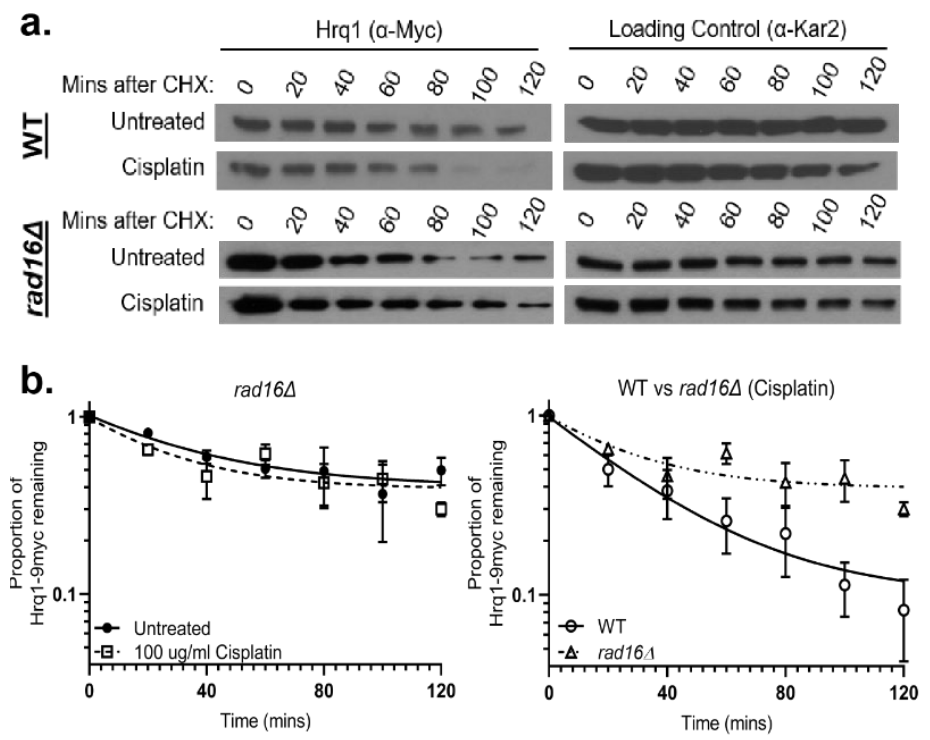

Figure 2. Hrq1 protein levels are stabilized in the absence of the E3 Ub-ligase, $R A D 16$. a)

Deletion of Rad16 stabilizes Hrq1 following cisplatin exposure. Hrq1-9xMYC expressing wildtype (WT) or rad16 6 , cells were incubated with cycloheximide in the presence or absence of 100 $\mu \mathrm{g} / \mathrm{ml}$ cisplatin and/or 0.1\% DMSO. Note blot from Figure 1c was reshown for comparison. b) Quantification of the proportion of Hrq1 remaining relative to time 0 (before CHX addition) and the loading control, Kar2, are plotted on the graph in log scale from WT and rad16 $\Delta$ cells. Each experiment was performed three to 5 times with standard error plotted. Note that the WT cisplatin treated time course is replotted from Figure 1c, for direct comparison to rad16 cisplatin treated cells. 
bioRxiv preprint doi: https://doi.org/10.1101/2022.03.01.482446; this version posted March 1,2022. The copyright holder for this preprin (which was not certified by peer review) is the author/funder, who has granted bioRxiv a license to display the preprint in perpetuity. It is made available under aCC-BY 4.0 International license.

\section{Figure 3}
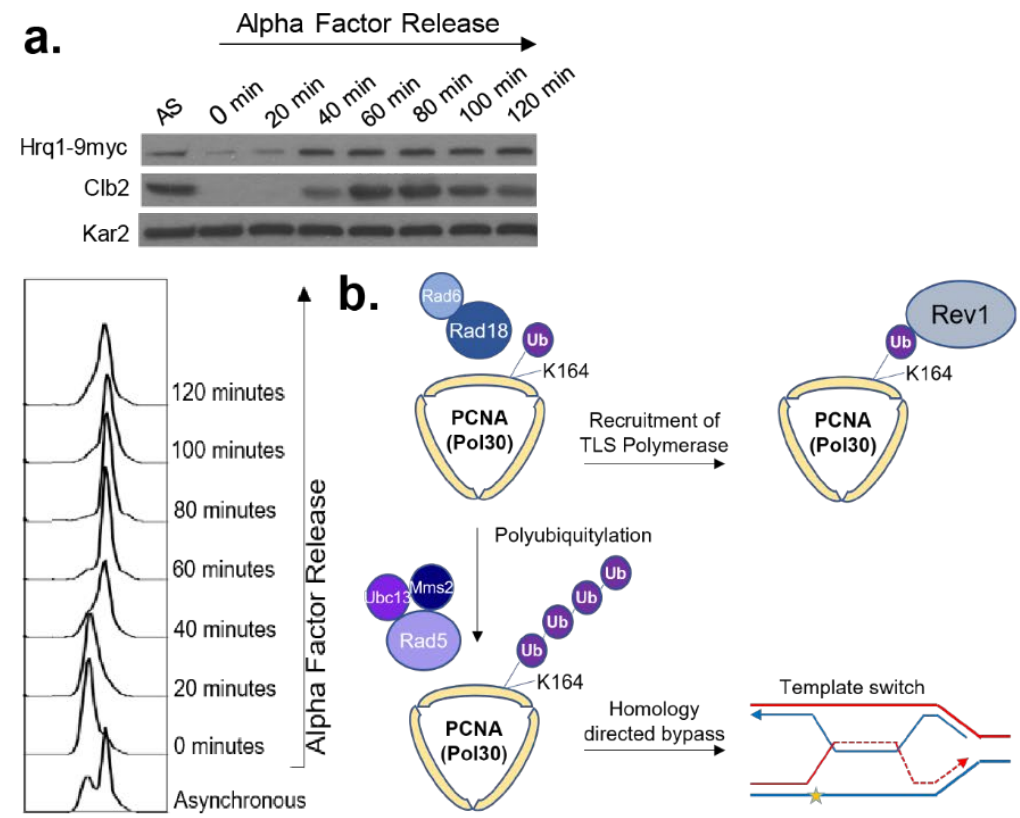

C.
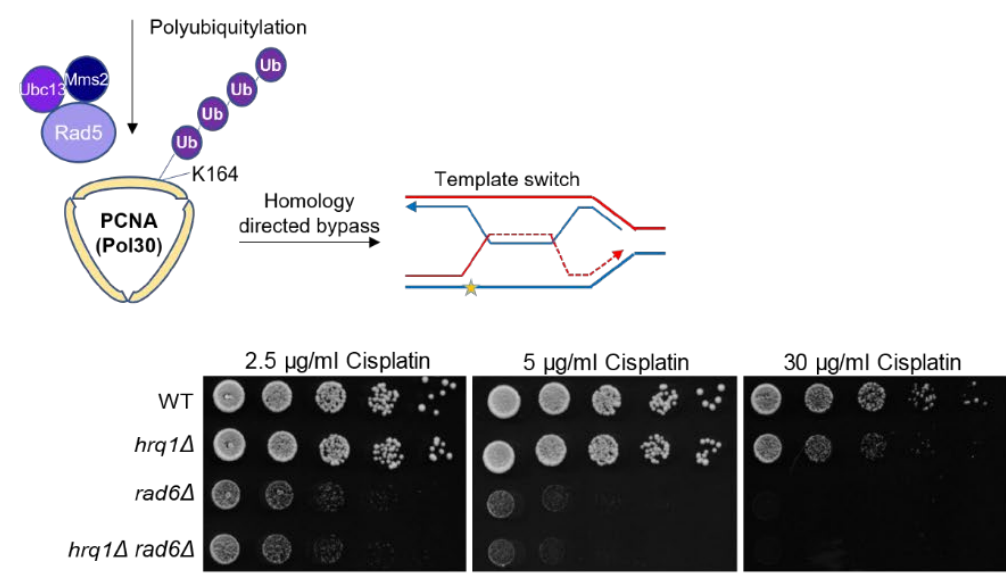

$0.1 \%$ DMSO

d.

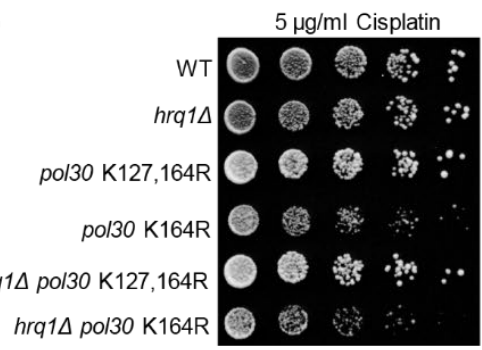

$10 \mu \mathrm{g} / \mathrm{ml}$ Cisplatin

$20 \mu \mathrm{g} / \mathrm{ml}$ Cisplatin

$30 \mu \mathrm{g} / \mathrm{ml}$ Cisplatin
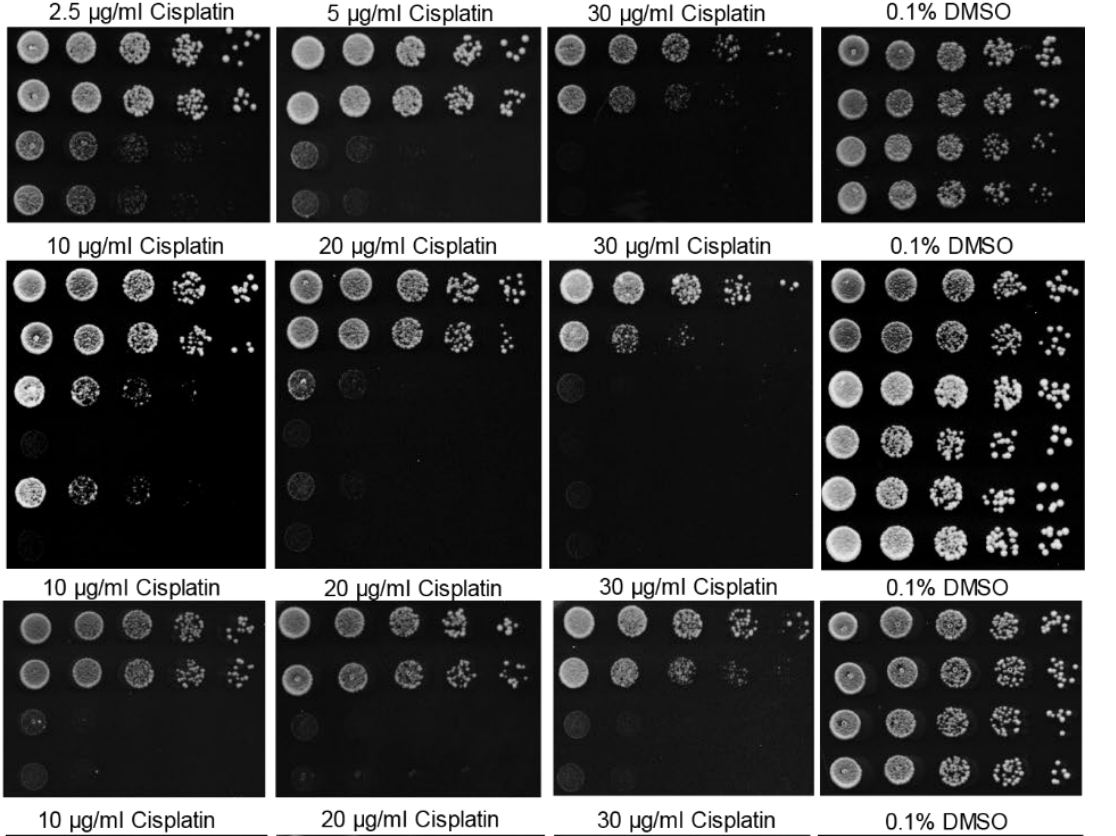

$0.1 \%$ DMSO

e.

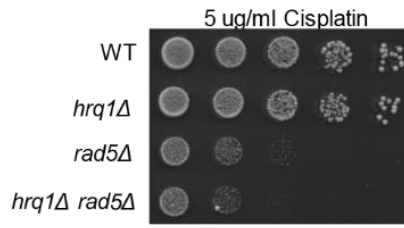

$10 \mu \mathrm{g} / \mathrm{ml}$ Cisplatin

$20 \mu \mathrm{g} / \mathrm{ml}$ Cisplatin
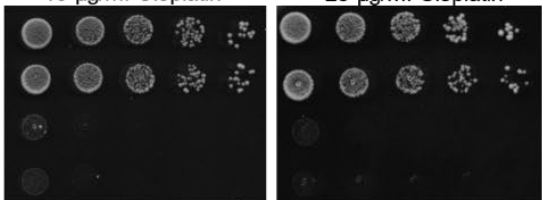

$30 \mu \mathrm{g} / \mathrm{ml}$ Cisplatin
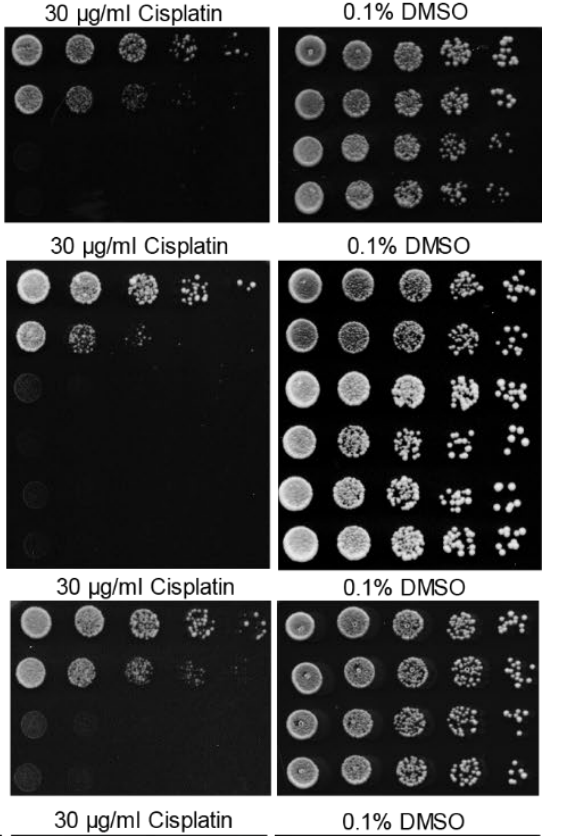

$0.1 \%$ DMSO

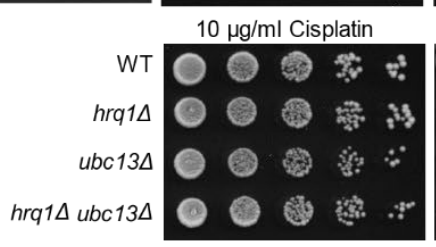

$20 \mu \mathrm{g} / \mathrm{ml}$ Cisplatin

$30 \mu \mathrm{g} / \mathrm{ml}$ Cisplatin

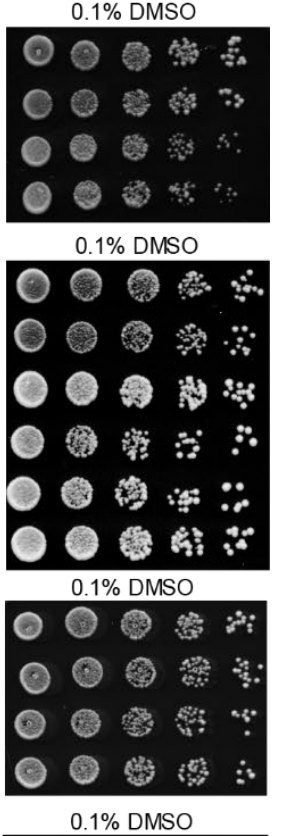

웅
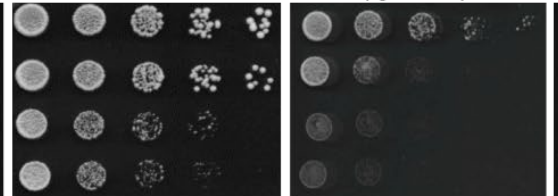

100 管

f.

$10 \mu \mathrm{g} / \mathrm{ml}$ Cisplatin

$20 \mu \mathrm{g} / \mathrm{ml}$ Cisplatin

$30 \mu \mathrm{g} / \mathrm{ml}$ Cisplatin $0.1 \%$ DMSO
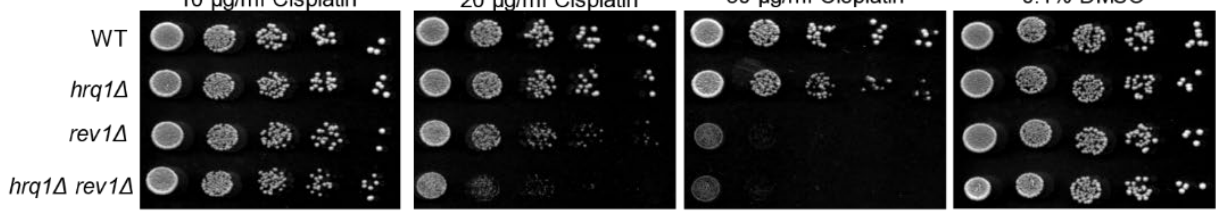
Figure 3. Hrq1 functions during error-free post-replicative repair. a) Hrq1 expression peaks during S/G2. Hrq1-9xMYC expressing cells were either untreated (asynchronous, AS) or cell cycle arrested in G1 with $\alpha$-factor. The $\alpha$-factor arrested cells were subsequently released into fresh YPD medium (0 min) and grown for $120 \mathrm{~min}$. Protein samples from the indicated time points were analyzed by western blot for Hrq1 (anti-MYC), the G2/M cyclin, Clb2, (anti-Clb2), or a loading control, Kar2 (anti-Kar2). The cell cycle stage was analyzed FACS. b) Schematic of post-replicative repair pathways. When the replication fork stalls, Pol30 (yellow trimer) is initially monoubiquitylated (Ub, purple circle) via Rad6-Rad18 (light and dark blue circles) on lysine 164 (K164). Monoubiquitylation of PCNA recruits the error-prone translesion synthesis polymerases, i.e. Rev1 (gray circle) to bypass the lesion. Alternatively, PCNA is further polyubiquitylated by Rad5-Ubc13-Mms2 (light, medium, and dark purple complex). Polyubiquitylation of PCNA mediates error-free repair through template switching, which is a homolog directed process. A bypass intermediate is shown with the newly synthesized DNA in red and the lesion as a yellow star. c) Hrq1 functions in the same pathway as Pol30. The indicated yeast strains were five-fold serial diluted onto SC medium containing DMSO and/or SC medium containing the indicated amount of cisplatin. The plates were photographed after 2 days of incubation at $30^{\circ} \mathrm{C}$ in the dark. d) Hrq1 functions in the same pathway as $\operatorname{Rad} 5$ and Ubc13. Serial dilutions of the indicated yeast strains were performed as described in c). e) Hrq1 functions in a different pathway than Rev1. Serial dilutions of the indicated yeast strains were performed as described in c). 


\section{Figure 4}

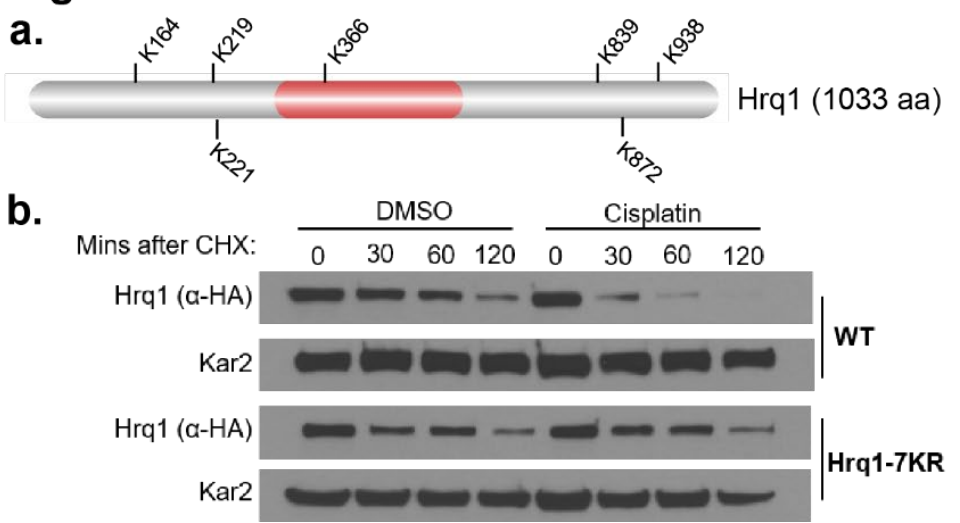

C.

Hrq1-7KR

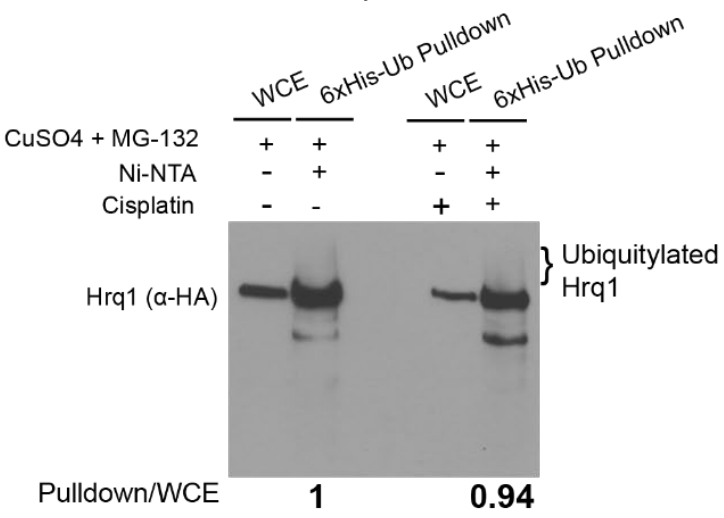

d.
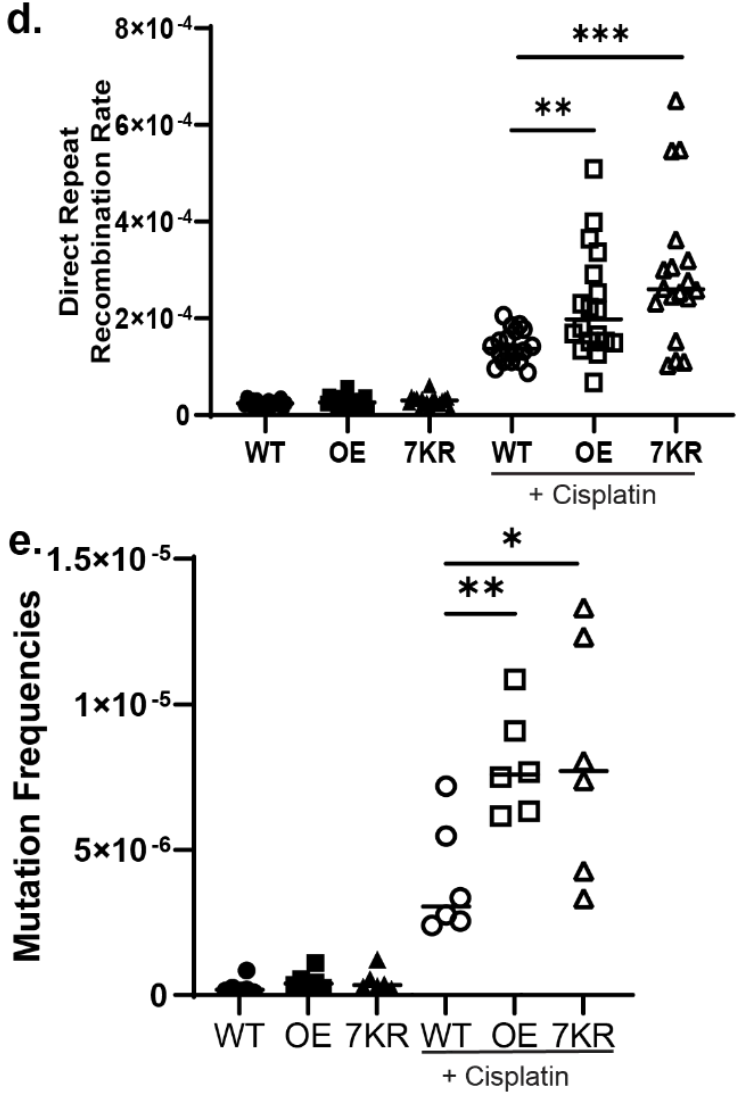
Figure 4. Hrq1 protein levels are stabilized following cisplatin exposure by mutating the predicted Hrq1 ubiquitylated lysine residues to arginine. a) Schematic of Hrq1 with lysines residues that are predicted to be ubiquitylated (K164, K219, K221, K872) or conserved between Hrq1 and RecQL4 (K366, K839, K938) is shown. The helicase domain is indicated in red (287496 aa, InterPro). b) Hrq1-7KR shows similar ubiquitylation in treated versus untreated conditions. Lanes 1 (whole cell extract, WCE without pulldown, 10\% input) serves as the control whereas lane 2 is the pulldown showing ubiquitylated Hrq1 as indicated. Lanes 3 and 4 is after treatment with cisplatin, where lane 3 is WCE and lane 4 is pulldown showing similar levels of ubiquitylated Hrq1 as untreated condition. c) Hrq1 protein levels are stabilized in Hrq1-7KR compared to WT. Cycloheximide chase experiments were performed in Hrq1-3xHA or Hrq17KR-6xHA expressing cells. Equal number of cells were collected every 30 minutes for 120 minutes. Experiment was performed in duplicate and a representative image (image was process using photoshop as described above) is shown. d) Overexpression or stabilization of Hrq1 leads to increased recombination following cisplatin treatment. Each measurement (dots) and the median value from three experiments (horizontal bar) were plotted. $* *$ represents $\mathrm{p}<0.01, * * *$ represents $\mathrm{p}<0.001 \mathrm{e})$ Overexpression or stabilization of Hrq1 leads to increased mutagenesis following cisplatin treatment. Each measurement (dots) and the median value from two experiments (horizontal bar) were plotted. * represents $\mathrm{p}<0.05, * *$ represents $\mathrm{p}<0.01$. 


\section{Figure 5}

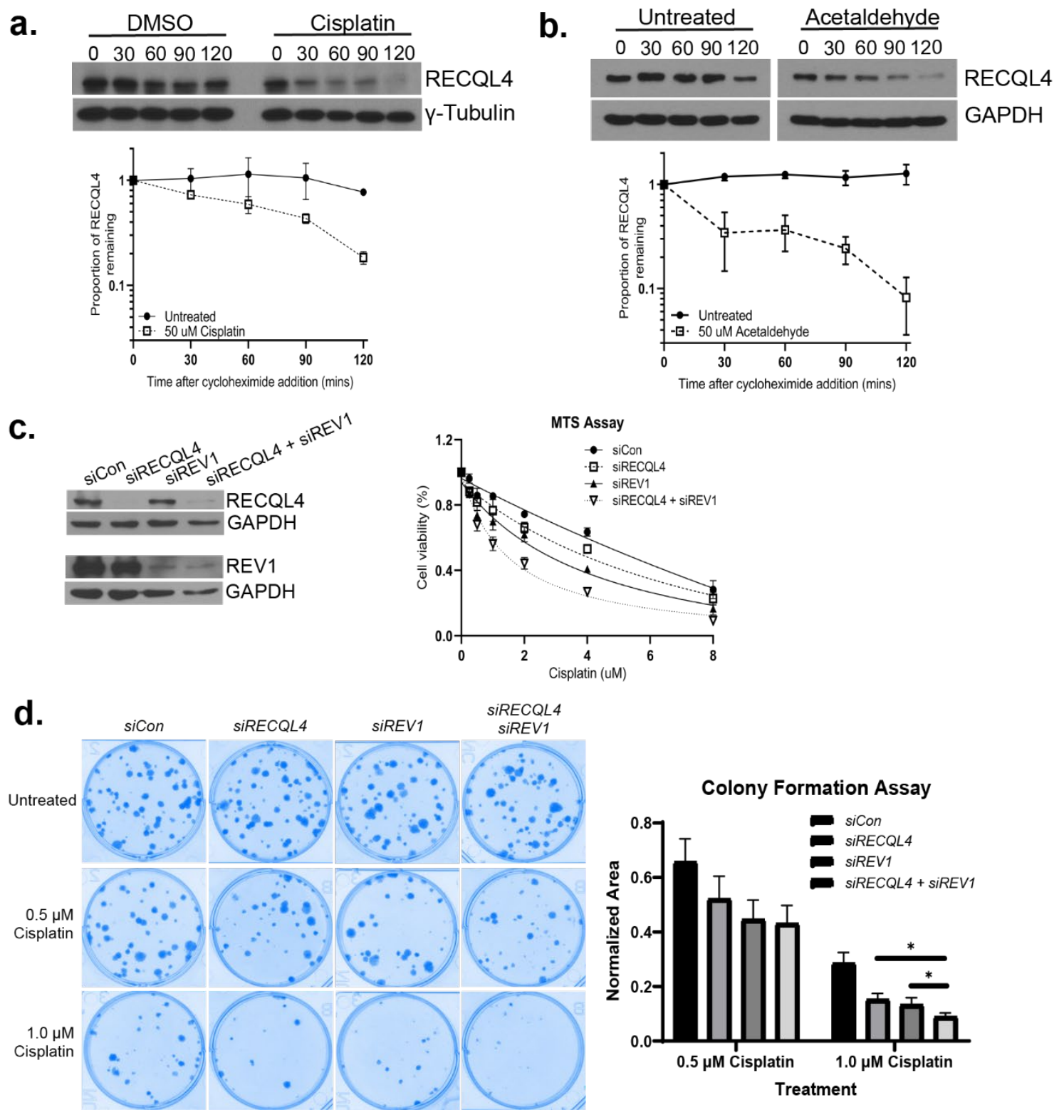

Figure 5. RECQL4 functions in a separate pathway from REV1 and is degraded following

DNA crosslinks. a) RECQL4 levels decrease following cisplatin treatment. U2OS cells were incubated with $50 \mu \mathrm{g} / \mathrm{ml}$ cycloheximide in the presence or absence of $50 \mu \mathrm{g} / \mathrm{ml}$ cisplatin. Protein extracts were analyzed by western blot for endogenous RECQL4 protein levels ( $\alpha$-RECQL4) or a loading control, Tubulin ( $\gamma$-Tubulin), at the indicated time points. Quantification of the proportion of RECQL4 remaining relative to time 0 (before $\mathrm{CHX}$ addition) and the loading 
control, Tubulin, are plotted on the graph in log scale. Each experiment was performed in triplicate with standard error plotted. b) RECQL4 protein levels decrease following acetaldehyde treatment. U2OS cells were incubated with $50 \mu \mathrm{g} / \mathrm{ml}$ cycloheximide in the presence or absence of $50 \mu \mathrm{g} / \mathrm{ml}$ acetaldehyde. Protein extracts were analyzed by western blot for endogenous RECQL4 protein levels ( $\alpha$-RECQL4) or a loading control, GAPDH ( $\alpha$-GAPDH), at the indicated time points. Quantification of the proportion of RECQL4 remaining relative to time 0 (before CHX addition) and the loading control, GAPDH, are plotted on the graph in log scale. Each experiment was performed in triplicate with standard error plotted. c) Cisplatin exposed U2OS cells exhibit decreased viability when both RECQL4 and REV1 are knocked down by siRNA. MTS assay was performed on after $72 \mathrm{hr}$ exposure to the indicated dose of cisplatin. Western is shown to confirmed knockdown of proteins, note since RECQL4 and REV1 are of similar size, the same samples were loaded twice to detect RECQL4 and REV1. Each experiment was performed three times with mean and standard errors plotted. d) Decreased cell viability following cisplatin exposure when both RECQL4 and REV1 are knocked down. Clonogenic survival assay with siControl (scrambled siCon), siRECQL4, siREV1, and siRECQL4 siREV1 treated cells with or without cisplatin. Each condition was plated in triplicate for each experiment and the experiment was performed three times with mean and standard errors plotted. 


\section{Figure 6}

a.

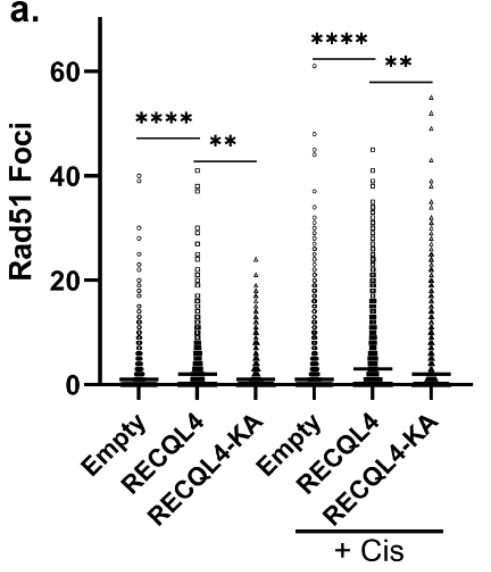

b.

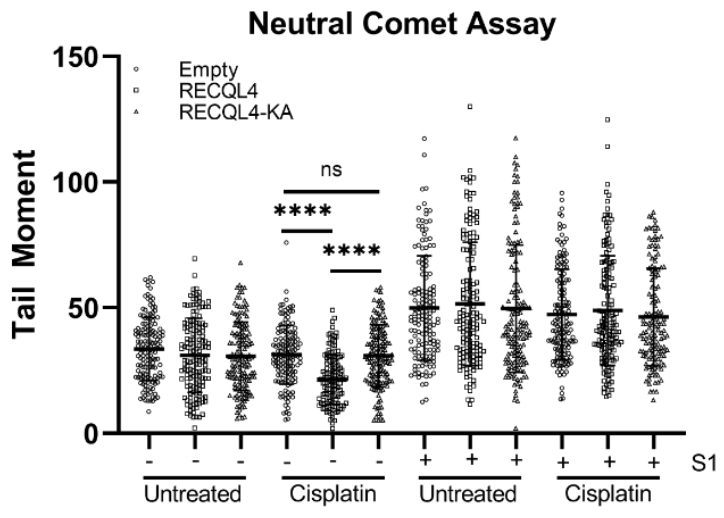

Figure 6. Overexpression of $R E C Q L 4$ results in increased RAD51 foci and decreased tail

moment. a) Overexpression of RECQL4 results in increased RAD51 foci, which is dependent on its helicase activity. U2OS cells were transfected with an empty plasmid or a plasmid expressing RECQL4 or RECQL4-K508A under a CMV promoter. The cells were either mock or cisplatin treated for one hour and after a two-hour recovery, imaged for RAD51 foci or DAPI by immunofluorescence. RAD51 foci was quantified from 200 cells per condition for each experiment. The experiment was performed three to five times and the median was graphed. Representative images are shown. b) Overexpression of RECQL4 results decreased tail moment following cisplatin exposure, which is dependent on its helicase activity. U2OS cells were treated similarly to the immunofluorescence experiment, before being harvested for neutral 
bioRxiv preprint doi: https://doi.org/10.1101/2022 03.01.482446; this version posted March 1, 2022. The copyright holder for this preprint (which was not certified by peer review) is the author/funder, who has granted bioRxiv a license to display the preprint in perpetuity. It is made available under aCC-BY 4.0 International license.

comet assay. At least 40 comets were counted per condition for each experiment. The experiment was performed four times and the mean and standard deviation was graphed. 
bioRxiv preprint doi: https://doi. org/10.1101/2022 03.01.482446; this version posted March 1, 2022 The copyright holder for this preprint (which was not certified by peer review) is the author/funder, who has granted bioRxiv a license to display the preprint in perpetuity. It is made available under aCC-BY 4.0 International license.

\section{Figure 7}

a.

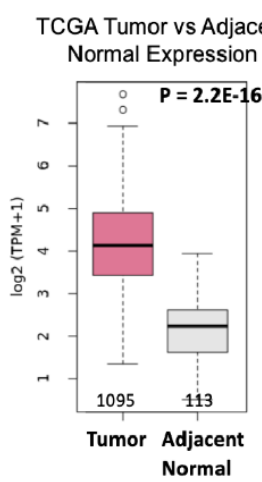

C.

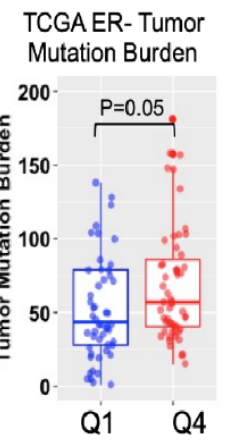

e.

Top20 Enriched DNA

Repair Pathway genes

\begin{tabular}{|c|c|c|}
\hline & Log2FC & padj \\
\hline RAD51 & 0.907 & $6.60 \mathrm{E}-06$ \\
\hline ZWINT & 0.876 & $5.80 E-46$ \\
\hline ZNF707 & 0.773 & $1.56 \mathrm{E}-41$ \\
\hline TYMS & 0.767 & $4.64 \mathrm{E}-54$ \\
\hline VPS28 & 0.657 & $3.44 \mathrm{E}-31$ \\
\hline ALYREF & 0.632 & $1.05 \mathrm{E}-46$ \\
\hline POLD1 & 0.622 & $2.74 \mathrm{E}-41$ \\
\hline FEN1 & 0.613 & $1.61 \mathrm{E}-43$ \\
\hline ADRM1 & 0.612 & $2.25 \mathrm{E}-33$ \\
\hline LIG1 & 0.595 & $1.76 \mathrm{E}-46$ \\
\hline SAC3D1 & 0.577 & $4.74 \mathrm{E}-45$ \\
\hline NME1 & 0.558 & $3.50 \mathrm{E}-30$ \\
\hline PCNA & 0.522 & $9.06 \mathrm{E}-21$ \\
\hline NME3 & 0.510 & $2.77 \mathrm{E}-27$ \\
\hline RFC4 & 0.474 & $1.19 \mathrm{E}-15$ \\
\hline POLR2K & 0.457 & $1.46 \mathrm{E}-27$ \\
\hline BRF2 & 0.438 & $2.80 \mathrm{E}-22$ \\
\hline NT5C & 0.425 & $9.43 \mathrm{E}-08$ \\
\hline POLA2 & 0.388 & $2.47 \mathrm{E}-16$ \\
\hline APRT & 0.385 & $1.04 \mathrm{E}-21$ \\
\hline
\end{tabular}

HR

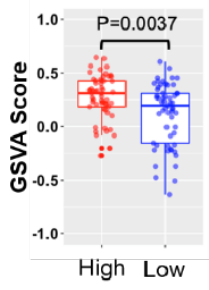

f.

g. b. RECQL4 Expression

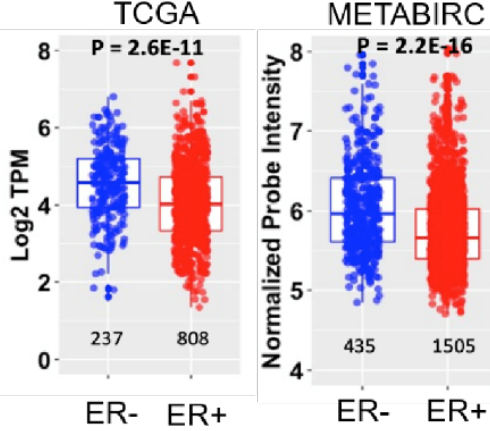

d.
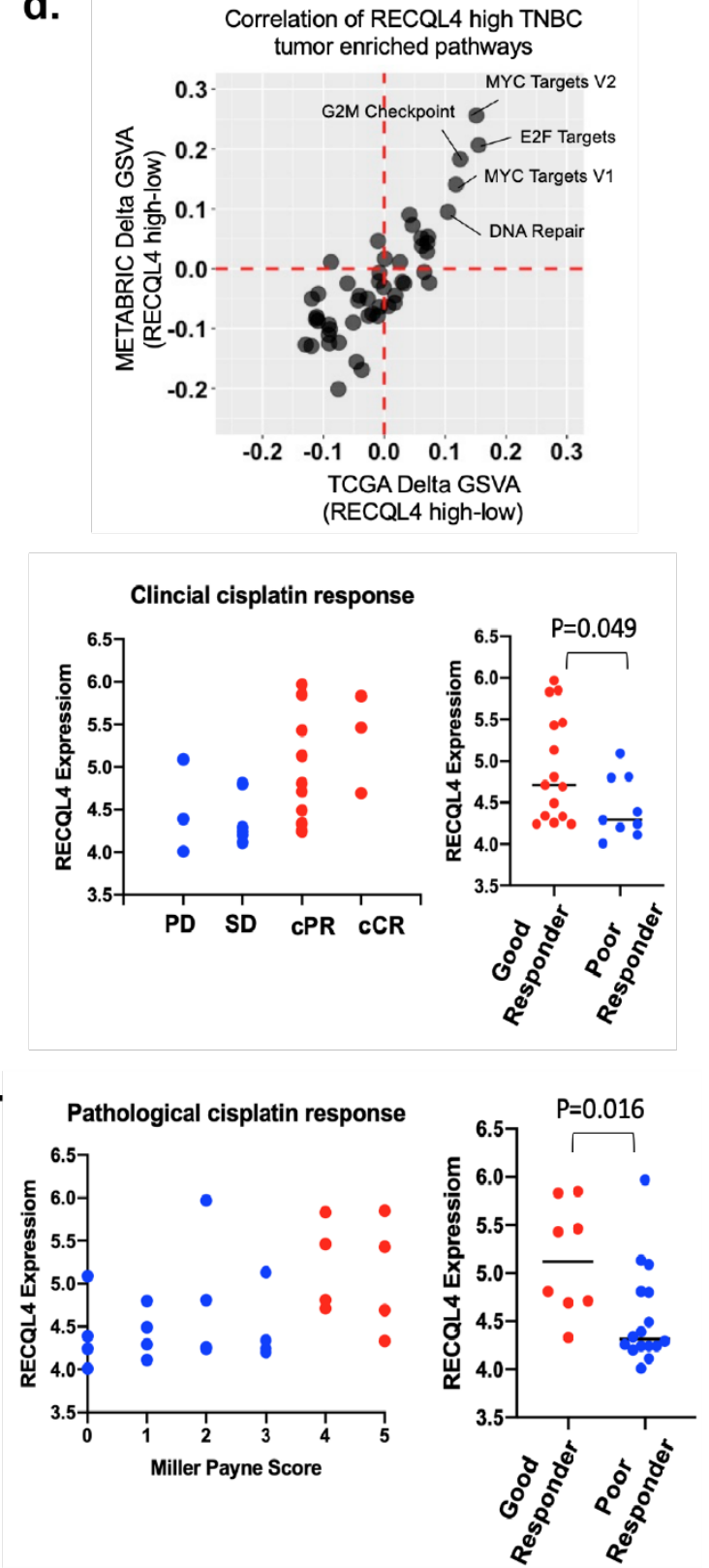
Figure 7. High RECQL4 expression correlates with increased recombination, mutations,

and tumorigenesis. a) RECQL4 expression is elevated in tumors in comparison to adjacent normal tissue. RNAseq data from tumors and normal tissue matched samples was acquired from TCGA. The data was normalized by transcripts per million (TPM), then analyzed for RECQL4 expression. Box and whisker plots graph the median of the data alongside the $25^{\text {th }}$ and $75^{\text {th }}$ percentile. The number of samples alongside p-value are shown using Mann-Whitney U test. b) RECQL4 expression is higher in ER- breast cancer. Transcriptome data from TCGA (RNA-seq) and METABRIC (microarray) was analyzed for RECQL4 expression in ER- (blue) and ER+ (red) cancers. Log2 (TPM+1) and normalized probe intensity were used respectively. Box and whisker plots alongside each data point was graphed. The number of samples alongside p-value are shown using Mann-Whitney U test. c) Elevated levels of RECQL4 are associated with increased tumor mutation burden. The expression of RECQL4 in ER- breast tumors from TCGA was divided into quartiles based upon expression level. The mutation burden between the least expressing tumors (Quartile 1-Q1, blue) and the highest expressing tumors (Quartile 4-Q4, red) was analyzed. Box and whisker plots alongside each data point was graphed. The number of samples alongside p-value are shown. Annotated mutational data from ER- breast cancer was acquired from Firebrower. The mutational data was categorized using "maftools" in R. Tumor mutation burden was scored as described (Wang et al 2019). Significance was determined by Mann-Whitney U test. d) High expression of RECQL4 correlates with enrichment of DNA repair, G2M checkpoint, MYC Targets V1, E2F Targets 1 and 2 in TNBC. Gene set variation analysis (GSVA) was performed on TNBC gene expression data from both TCGA and METABRIC using the 50 Hallmark gene sets from MSigDB. Correlation between TCGA and METABRIC datasets was plotted for enriched pathways. e) Homologous recombination and 
RAD51 is enriched in TNBC tumors expressing high levels of RECQL4. GVSA results of “KEGG Homologous Recombination” gene set (MSigDB M11675) was parsed for the top 20 DNA repair genes enriched in TNBC tumors expressing high levels of RECQL4. HR pathway was enriched by GSVA analysis in RECQL4 high expressing TNBC compared to loss expressing TBNC. Box and whisker plots alongside each data point was graphed. The number of samples alongside p-value are shown using Mann-Whitney U test. f, g) RECQL4 expression serves as a predictor for clinical outcomes. High expression of RECQL4 correlates with positive response to cisplatin and favorable clinical outcome (Good responder). Analysis from a (Silver et al 2010) study where cisplatin was given as a neoadjuvant therapy in 24 TNBC tumors. Lower expressing RECQL4 tumors are shown as blue dots and high expressing RECQL4 tumors are shown as red dots. Clinical response to cisplatin was measured as progressive disease (PD), stable disease (SD), partial response (cPR), complete response (cCR). Good and poor responders were defined as patients with $\mathrm{cPR} / \mathrm{cCR}$ status and $\mathrm{PD} / \mathrm{SD}$ status respectively. Increased RECQL4 expression correlates with a good clinical response as determined by Mann-Whitney U test. Pathological response to cisplatin was monitored by Miller Payne metric score (grade 1 is no significant tumor reduction and grade 5 is compete tumor reduction) and graphed based on RECQL4 expression. Good and poor responders were defined as patients with a Miller Payne Score of 4-5 or 0-3 respectively. Increased RECQL4 expression correlates with a good pathological response as determined by Mann-Whitney U test. 


\section{Figure 8}

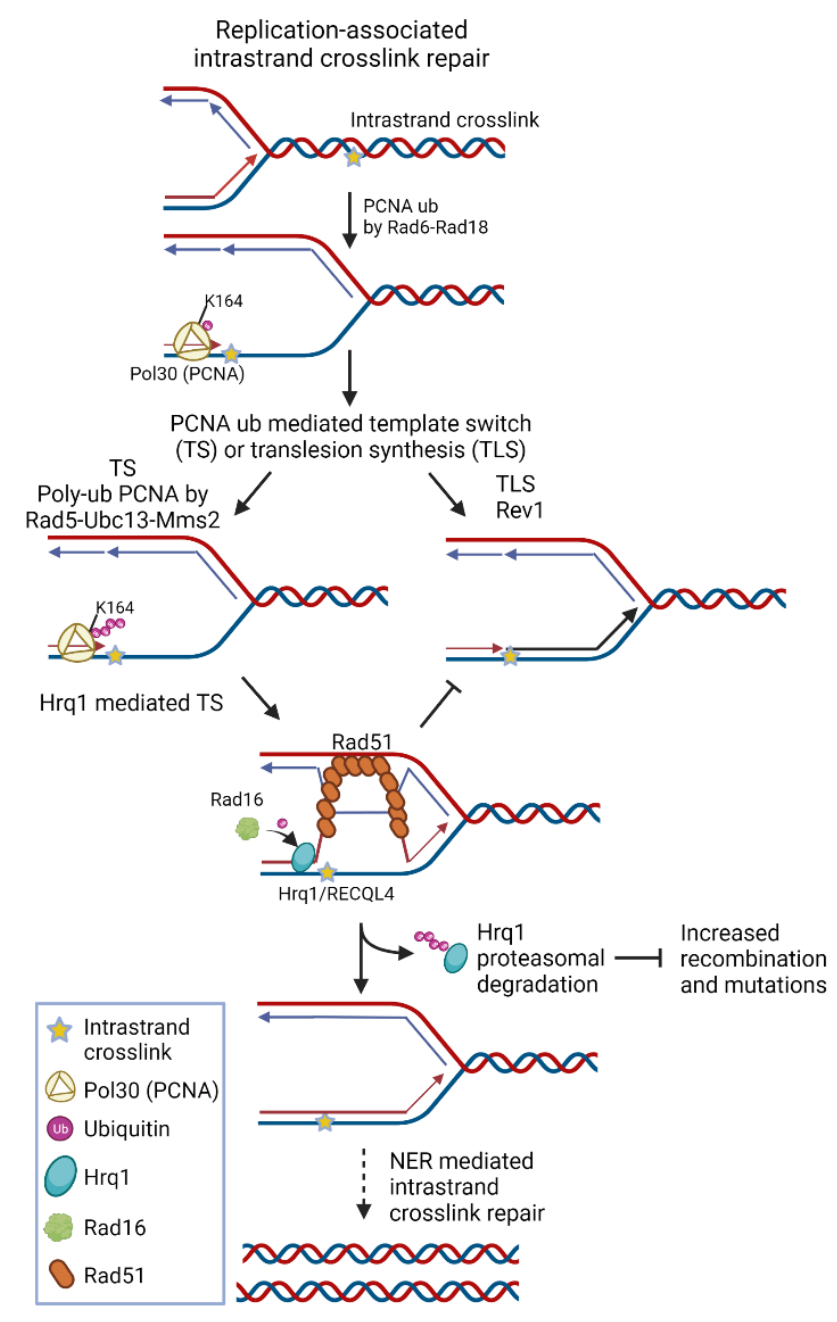

Figure 8. Model of Hrq1/RECQL4 function during replication-associated intrastrand

crosslink repair. When the replication fork stalls, PCNA (yellow trimer) is monoubiquitylated (Ub, purple circle) by Rad6-Rad18 on lysine 164 (K164). Monoubiquitylation of PCNA recruits the error-prone translesion synthesis polymerases, i.e. Rev1 to bypass the lesion. Alternatively, PCNA is further poly-ubiquitylated by Rad5-Ubc13-Mms2. Polyubiquitylation of PCNA mediates error-free repair through template switching, which is a homology directed process. Hrq1/RECQL4 (blue oval) facilitates a template switch to bypass the lesion and then is subsequently ubiquitylated by Rad16 and degraded. Degradation of Hrq1 by the proteasome 
bioRxiv preprint doi: https://doi.org/10.1101/2022.03.01.482446; this version posted March 1, 2022. The copyright holder for this preprint (which was not certified by peer review) is the author/funder, who has granted bioRxiv a license to display the preprint in perpetuity. It is made available under aCC-BY 4.0 International license.

prevents aberrant recombination and subsequent mutation. Once the lesion is bypass, the NER machinery facilitates repair. Created using biorender.com 